\title{
BOUNDARY OBSERVABILITY FOR THE SPACE SEMI-DISCRETIZATIONS OF THE 1 - D WAVE EQUATION
}

\author{
Juan Antonio Infante ${ }^{1}$ And EnRique ZuazuA ${ }^{1, *}$
}

\begin{abstract}
We consider space semi-discretizations of the $1-d$ wave equation in a bounded interval with homogeneous Dirichlet boundary conditions. We analyze the problem of boundary observability, i.e., the problem of whether the total energy of solutions can be estimated uniformly in terms of the energy concentrated on the boundary as the net-spacing $h \rightarrow 0$. We prove that, due to the spurious modes that the numerical scheme introduces at high frequencies, there is no such a uniform bound. We prove however a uniform bound in a subspace of solutions generated by the low frequencies of the discrete system. When $h \rightarrow 0$ this finite-dimensional spaces increase and eventually cover the whole space. We thus recover the well-known observability property of the continuous system as the limit of discrete observability estimates as the mesh size tends to zero. We consider both finite-difference and finite-element semi-discretizations.

Résumé. On considère l'approximation par différences finies et éléments finis en espace de l'équation des ondes $1-d$ avec des conditions aux limites de Dirichlet homogènes. On étudie le problème de l'observabilité frontière, i.e., la possibilité d'estimer l'énergie totale des solutions par l'énergie concentrée sur un extrême du bord, uniformement lorsque $h$, le pas de la discrétisation, tend vers zéro. On démontre que cette estimation uniforme n'a pas lieu à cause d'un comportement singulier des fonctions propres à hautes fréquences. Néanmoins, on démontre une estimation uniforme dans des sous-espaces convenables de solutions qui, lorsque $h \rightarrow 0$, finissent par couvrir l'espace d'énergie tout entier. On retrouve donc la propriété d'observabilité, bien connue pour le système continu, comme la limite des estimations discrètes lorsque le pas en espace tend vers zéro.
\end{abstract}

AMS Subject Classification. 35L05, 65M06, 65M60, 93B07.

Received: March 7, 1998. Revised: July 10, 1998.

\section{Introduction: Finite-Difference SEMI-DisCREtizations}

Consider the $1-d$ wave equation

$$
\left\{\begin{array}{l}
u_{t t}-u_{x x}=0, \quad 0<x<L, \quad 0<t<T \\
u(0, t)=u(L, t)=0, \quad 0<t<T \\
u(x, 0)=u_{0}(x), \quad u_{t}(x, 0)=u_{1}(x), \quad 0<x<L .
\end{array}\right.
$$

Keywords and phrases. Wave equation, semi-discretization, observability.

${ }^{1}$ Universidad Complutense, Matematica Aplicada, 28040 Madrid, Spain. e-mail: infante@sunma4.mat.ucm.es, zuazua@sunma4.mat.ucm.es

* This work was supported by grant PB96-0663 of the DGES (Spain) and ERB-FMRX-CT960033 of the EU. 
System (1.1) is well-posed in the energy space $H_{0}^{1}(0, L) \times L^{2}(0, L)$. More precisely, for any $\left(u_{0}, u_{1}\right) \in H_{0}^{1}(0, L) \times$ $L^{2}(0, L)$ there exists a unique solution $u \in C\left([0, T] ; H_{0}^{1}(0, L)\right) \cap C^{1}\left([0, T] ; L^{2}(0, L)\right)$.

The energy of solutions is given by

$$
E(t)=\frac{1}{2} \int_{0}^{L}\left[\left|u_{t}(x, t)\right|^{2}+\left|u_{x}(x, t)\right|^{2}\right] d x
$$

and it is conserved along time, i.e.

$$
E(t)=E(0), \quad \forall 0<t<T .
$$

It is by now well known that when $T>2 L$, the total energy of solutions can be estimated uniformly by means of the energy concentrated on one extreme of the boundary, say, $x=L$. More precisely, for any $T>2 L$ there exists $C(T)>0$ such that

$$
E(0) \leq C(T) \int_{0}^{T}\left|u_{x}(L, t)\right|^{2} d t
$$

for every finite energy solution of (1.1).

When the energy concentrated on the boundary is measured in both extremes $x=0$ and $L$, the inequality holds for all $T>L$.

In this paper we focus on inequality (1.4).

Inequalities of the form (1.4) are related to the boundary controllability of the wave equation. We refer to [9] and [11] for a systematic analysis of these issues, both in the context of wave equations and plate models.

In this paper we analyze the analogue of (1.4) for several space semi-discretizations of the wave equation.

Let us consider first the finite-difference semi-discretization to illustrate the kind of problems we have in mind.

Given $N \in N$ we set $h=L /(N+1)$ and introduce the net

$$
x_{0}=0<x_{1}=h<\cdots<x_{N}=N h<x_{N+1}=L
$$

with $x_{j}=j h, j=0, \cdots, N+1$.

We then introduce the following finite-difference semi-discretization of (1.1)

$$
\left\{\begin{array}{l}
u_{j}^{\prime \prime}(t)=\frac{\left[u_{j+1}(t)+u_{j-1}(t)-2 u_{j}(t)\right]}{h^{2}}, 0<t<T, j=1, \cdots, N \\
u_{0}(t)=u_{N+1}(t)=0, \quad 0<t<T \\
u_{j}(0)=u_{j}^{0}, u_{j}^{\prime}(0)=u_{j}^{1}, \quad j=0, \cdots, N+1
\end{array}\right.
$$

In $(1.6)^{\prime}$ denotes derivation with respect to time.

System (1.6) is a system of $N$ linear differential equations with $N$ unknowns $u_{1}, \cdots, u_{N}$, since, in view of the boundary conditions, $u_{0} \equiv u_{N+1} \equiv 0$.

Obviously, $u_{j}(t)$ is an approximation of $u\left(x_{j}, t\right), u$ being the solution of (1.1), provided the initial data $\left(u_{j}^{0}, u_{j}^{1}\right), j=0, \cdots, N+1$ are an approximation of the initial data in (1.1).

The energy of system (1.6) is given by

$$
E_{h}(t)=\frac{h}{2} \sum_{j=0}^{N}\left[\left|u_{j}^{\prime}(t)\right|^{2}+\left|\frac{u_{j+1}(t)-u_{j}(t)}{h}\right|^{2}\right]
$$

which is a discretization of the continuous energy $E$. 
It is easy to see that the energy $E_{h}$ is conserved along time for the solutions of (1.6), i.e.

$$
E_{h}(t)=E_{h}(0), \quad \forall 0<t<T .
$$

The main goal of this paper is to analyze the following discrete version of (1.4):

$$
E_{h}(0) \leq C(T, h) \int_{0}^{T}\left|\frac{u_{N}(t)}{h}\right|^{2} d t
$$

Remark 1.1. Let us discuss the choice of the approximation $-u_{N}(t) / h$ for the normal derivative $u_{x}(L, t)$. Needless to say, Taylor's expansion suggests that the simplest approximation for $u_{x}(L, t)$ is

$$
\frac{u(L, t)-u(L-h, t)}{h}
$$

or, with the notation above,

$$
u_{x}(L, t) \sim \frac{u_{N+1}(t)-u_{N}(t)}{h} .
$$

Taking into account that, due to the Dirichlet boundary conditions, $u_{N+1}=0$, we deduce that

$$
u_{x}(L, t) \sim \frac{-u_{N}(t)}{h} .
$$

On the other hand, as we shall see in Section 2.1, it follows that $-u_{N} / h \rightarrow u_{x}(L)$ as $h \rightarrow 0$ for each eigenfunction when the frequency is fixed. This indicates that $-u_{N}(t) / h$ is also a good approximation for the solutions of the wave equation (1.1) (see also Remark 2.1).

In view of (1.4) one may expect that, when $T>2 L$, there exists $C=C(T)>0$ independent of $h$ such that (1.9) holds for every solution of (1.6) and for every $0<h<1$.

The first result of this paper asserts that this is false:

Theorem 1.1. For any $T>0$, we have

$$
\sup _{u \text { solution of }(1.6)}\left[\frac{E_{h}(0)}{\int_{0}^{T}\left|u_{N}(t) / h\right|^{2} d t}\right] \rightarrow \infty \text { as } h \rightarrow 0 .
$$

As we shall see, this is due to the spurious modes that the numerical scheme introduces at high frequencies. This was already observed by R. Glowinski et al. in [3-5], in connection with the exact boundary controllability of the wave equation in several space dimensions and the numerical implementation of the so-called HUM method (see J.L. Lions [11]). In these works two methods were proposed to cure this high frequency pathology: (a) A Tychonoff regularization procedure for the quadratic functional to be minimized when computing the controls; (b) A filtering technique to eliminate the short wave length components of the solutions of the discrete system. The efficiency of both methods was exhibited in these works by various numerical experiments.

To prove Theorem 1.1 we analyze the spectrum of (1.6) and we use discrete multiplier techniques to derive sharp observability inequalities for the eigenvectors of the eigenvalue problem associated to (1.6). In order to prove the positive counterpart of Theorem 1.1, i.e. inequalities of the form (1.9) which are uniform as $h \rightarrow 0$, we use discrete multiplier techniques. As we mentioned above, in order for these inequalities to be uniform, one has to rule out the high frequency spurious modes introduced by the numerical scheme. This will be done by considering suitable classes of solutions of (1.6) generated by the low frequency eigenvectors of (1.6), or, in other words, by a suitable truncation of the Fourier development of solutions of (1.6). Thus, our approach is 
very close to the filtering technique mentioned above (we refer to Glowinski [3] for a complete discussion of this issue).

To make our statements precise, let us consider the eigenvalue problem associated with (1.6):

$$
\left\{\begin{array}{l}
-\left[\frac{\varphi_{j+1}+\varphi_{j-1}-2 \varphi_{j}}{h^{2}}\right]=\lambda \varphi_{j}, \quad j=1, \cdots, N \\
\varphi_{0}=\varphi_{N+1}=0 .
\end{array}\right.
$$

Let us denote by $\lambda_{1}(h), \cdots, \lambda_{N}(h)$ the $N$ eigenvalues of (1.11):

$$
0<\lambda_{1}(h)<\lambda_{2}(h)<\cdots<\lambda_{N}(h) .
$$

These eigenvalues can be computed explicitly. We have (see [8], p. 456):

$$
\lambda_{k}(h)=\frac{4}{h^{2}} \sin ^{2}\left(\frac{\pi k h}{2 L}\right), k=1, \cdots, N .
$$

The eigenfunction $\varphi^{k}=\left(\varphi_{k, 1}, \cdots, \varphi_{k, n}\right)$ associated to the eigenvalue $\lambda_{k}(h)$ can also be computed explicitly:

$$
\varphi_{k, j}=\sin \left(j \frac{\pi h k}{L}\right), j=1, \cdots, N
$$

Solutions of (1.6) admit a Fourier development on the basis of eigenvectors of system (1.11). More precisely, every solution $u=\left(u_{1}, \cdots, u_{N}\right)$ of $(1.6)$ can be written as

$$
u(t)=\sum_{k=1}^{N}\left[a_{k} \sin \left(\sqrt{\lambda_{k}(h)} t\right)+b_{k} \cos \left(\sqrt{\lambda_{k}(h)} t\right)\right] \varphi^{k}
$$

for suitable coefficients $a_{k}, b_{k} \in \mathbb{R}, k=1, \cdots, N$, that can be computed explicitly in terms of the initial data in (1.6).

Before getting into the discussion of the observability of solutions of (1.6) it is interesting to analyze the boundary observability of the eigenvectors. The following Lemma provides the answer:

Lemma 1.1. For any eigenvector $\varphi=\left(\varphi_{1}, \cdots, \varphi_{N}\right)$ of system (1.11) the following identity holds:

$$
h \sum_{j=0}^{N}\left|\frac{\varphi_{j+1}-\varphi_{j}}{h}\right|^{2}=\frac{2 L}{4-\lambda h^{2}}\left|\frac{\varphi_{N}}{h}\right|^{2} .
$$

This identity provides an explicit relation between the total energy of the eigenvectors (the left hand side of (1.16)) and the energy concentrated on the extreme $x=L$ which is represented by the quantity $\left|\varphi_{N} / h\right|^{2}$.

On the other hand, it is easy to check that

$$
\lambda h^{2}<4
$$

for all $h>0$ and all the eigenvalues of (1.11). But, obviously, (1.17) does not exclude the blow up of the constant in the right hand side of (1.16). In fact, one can check that

$$
\lambda_{N}(h) h^{2} \rightarrow 4 \text { as } h \rightarrow 0 .
$$

Therefore blow-up occurs. This immediately yields the negative result of Theorem 1.1. 
In order to obtain a positive counterpart to Theorem 1.1 we have to introduce suitable subclasses of solutions of (1.6). Given any $0<\gamma<4$ we introduce the class $\mathcal{C}_{h}(\gamma)$ of solutions of (1.6) generated by eigenvectors of (1.11) associated with eigenvalues such that

$$
\lambda h^{2} \leq \gamma
$$

More precisely,

$$
\mathcal{C}_{h}(\gamma):=\left\{u=\sum_{\lambda_{k}(h) \leq \gamma h^{-2}}\left[a_{k} \sin \left(\sqrt{\lambda_{k}(h) t}\right)+b_{k} \cos \left(\sqrt{\lambda_{k}(h) t}\right)\right] \varphi^{k} \text { with } a_{k}, b_{k} \in \mathbb{R}\right\}
$$

According to Lemma 1.1, the energy of every eigenvector entering in $\mathcal{C}_{h}(\gamma)$ can be estimated uniformly in terms of the energy concentrated on the boundary.

The following result guarantees that this is in fact the case for all solutions of $(1.6)$ in the class $\mathcal{C}_{h}(\gamma)$ provided the length $T$ of the time interval is large enough:

Theorem 1.2. Assume that $0<\gamma<4$. Then, there exists $T(\gamma) \geq 2 L$ such that for all $T>T(\gamma)$ there exists $C=C(T, \gamma)$ such that (1.9) holds for every solution of (1.6) in the class $\mathcal{C}_{h}(\gamma)$, uniformly as $h \rightarrow 0$.

Moreover,

(a) $T(\gamma) \nearrow \infty$ as $\gamma \nearrow 4$ and $T(\gamma) \searrow 2 L$ as $\gamma \searrow 0$.

(b) $C(T, \gamma) \searrow \frac{L}{2(T-2 L)}$ as $\gamma \searrow 0$.

Remark 1.2. Theorem 1.2 asserts that the uniform observability inequality (1.9) holds in the class $\mathcal{C}_{h}(\gamma)$ provided $T$ is large enough. In fact, $T(\gamma) \rightarrow \infty$ as $\gamma \rightarrow 4$. This is due to the fact that the gap between the roots of consecutive eigenvalues vanishes as they approach the critical value. However, as $\gamma \rightarrow 0$ the observability time $T(\gamma)$ converges to $2 L$, which is the observability time for system (1.1). Note that, according to this result, the uniform observability inequality (1.9) holds for $T>2 L$ for solutions of (1.6) of the form

$$
u=\sum_{\lambda_{k}(h) \leq \mu(h)}\left[a_{k} \sin \left(\sqrt{\lambda_{k}(h)} t\right)+b_{k} \cos \left(\sqrt{\lambda_{k}(h)} t\right)\right] \varphi^{k}
$$

with $\mu(h)$ such that

$$
\mu(h) h^{2} \rightarrow 0 \text { as } h \rightarrow 0
$$

This allows to recover the observability of the original system (1.1) as the limit as $h \rightarrow 0$ of the observability of solutions of the form (1.21)-(1.22) of the semi-discrete system (1.6).

We also observe that the constant $C(T, \gamma)$ of the observability inequality (1.9) converges to $L / 2(T-2 L)$, which is the constant that one obtains by multiplier techniques for the observability of the continuous system (1.1) (see [11]).

Remark 1.3. It is easy to see that system (1.6) is observable. Since it is a system of ode's, we deduce that it is observable for all $T>0$ (see [10]). Therefore, for any $h>0$ and $T>0$ there exits $C(T, h)>0$ such that (1.9) holds for any solution of (1.6). However, in order to get a uniform (as $h \rightarrow 0$ ) observability constant we need to filter the high frequencies (i.e., to consider solutions in the class $\mathcal{C}_{h}(\gamma)$ ) and to take $T$ large enough. $\square$

Roughly speaking, Theorem 1.2 guarantees that the semi-discrete systems are uniformly observable as $h \rightarrow 0$ provided the high frequencies are filtered.

We shall give two proofs of Theorem 1.2. The first one is an adaptation of the classical multiplier techniques that are used to prove the observability of wave and plate equations (see $[9,11]$ ). The second one is based on the classical inequality by Ingham [7] for non-harmonic Fourier series. 
One may think that these results are due to the particular finite-difference discretization we have considered. But this is not the case. We also consider the finite-element space semi-discretization and obtain similar results, both, in what concerns the negative result of Theorem 1.1 but also the positive one of Theorem 1.2.

It is also worth mentioning that the phenomena we have described here for the discretizations of the wave equation have been found earlier in the context of the observability of the $1-d$ wave equation with rapidly oscillating periodic coefficients (see [1] and [2]). Roughly, in both cases, the interaction of waves with the microstructure or the discrete mesh produces spurious high frequency vibrations that are not observed in the limit continuous model.

The rest of the paper is organized as follows. Section 2 is devoted to the analysis of the finite-difference approximation. In particular, we develop and prove the results stated in this introduction. Section 3 is dedicated to the analysis of the finite-element discretization. In Section 4 we briefly compare finite-difference and finiteelement semi-discretizations.

\section{Finite-DifFEREnCE SEMI-DisCREtizAtion}

In this section we analyze in detail the problem of the observability of the finite-difference space semidiscretization (1.6) of the wave equation (1.1) that we have discussed in the introduction.

First of all we perform a careful analysis of the spectrum. In particular we prove Lemma 1.1 and, as an immediate consequence of it, Theorem 1.1. We then prove Theorem 1.2 in detail using multiplier techniques. We also indicate how the same results can be recovered using well-known results on non-harmonic Fourier series.

\subsection{Spectral analysis}

Let us recall the system that eigenfunctions $\varphi=\left(\varphi_{1}, \cdots, \varphi_{N}\right)$ and eigenvalues $\lambda$ of system (1.6) satisfy:

$$
\left\{\begin{array}{l}
-\left[\frac{\varphi_{j+1}+\varphi_{j-1}-2 \varphi_{j}}{h^{2}}\right]=\lambda \varphi_{j}, j=1, \cdots, N \\
\varphi_{0}=\varphi_{N+1}=0 .
\end{array}\right.
$$

This system is the eigenvalue problem of the matrix

$$
A=\frac{1}{h^{2}}\left(\begin{array}{ccccc}
2 & -1 & 0 & & 0 \\
-1 & 2 & -1 & & \\
\cdot & \cdot & \cdot & \cdot & \cdot \\
& & -1 & 2 & -1 \\
\mathbf{0} & & & -1 & 2
\end{array}\right) .
$$

The eigenvalues and eigenvectors of system (2.1) (or, of matrix $A$ ) can be computed explicitly. We have (see [8], p. 456):

$$
\lambda_{k}(h)=\frac{4}{h^{2}} \sin ^{2}\left(\frac{\pi k h}{2 L}\right), j=1, \cdots, N
$$

and

$$
\varphi^{k}=\left(\varphi_{k, j}\right)=\sin \left(\frac{j \pi h k}{L}\right), k=1, \cdots, N, j=1, \cdots, N
$$

Observe in particular that eigenvectors of the discrete system coincide with the eigenfunctions $\sin \left(\frac{\pi k x}{L}\right)$ of the continuous one. On the other hand, for $k$ fixed,

$$
\lambda_{k}(h) \rightarrow \frac{\pi^{2} k^{2}}{L^{2}}, \text { as } h \rightarrow 0
$$


which is the $k$ th eigenvalue of the continuous system.

Lemma 2.1. For any eigenvector $\varphi$ with eigenvalue $\lambda$ of (2.1) the following identity holds:

$$
\sum_{j=0}^{N}\left|\frac{\varphi_{j}-\varphi_{j+1}}{h}\right|^{2}=\lambda \sum_{j=1}^{N}\left|\varphi_{j}\right|^{2}
$$

If $\varphi^{k}$ and $\varphi^{\ell}$ are eigenvectors associated to eigenvalues $\lambda_{k} \neq \lambda_{\ell}$ it follows that

$$
\sum_{j=0}^{N}\left(\varphi_{k, j}-\varphi_{k, j+1}\right)\left(\varphi_{\ell, j}-\varphi_{\ell, j+1}\right)=0
$$

Proof. Multiplying in (2.1) by $\varphi_{j}$ and adding for $j=1, \cdots, N$ the identity (2.5) follows immediately. (Note that $(2.5)$ can be read as $(A \varphi, \varphi)=\lambda(\varphi, \varphi))$.

In order to get (2.6) we point out that, $A$ being symmetric, eigenvectors associated with distinct eigenvalues are orthogonal and $A$-orthogonal, i.e., $\left(\varphi^{k}, \varphi^{\ell}\right)=0$ and $\left(A \varphi^{k}, \varphi^{\ell}\right)=0$. Consequently

$$
\sum_{j=1}^{N} \varphi_{k, j} \varphi_{\ell, j}=0
$$

and

$$
0=\sum_{j=1}^{N}\left(\varphi_{k, j+1}+\varphi_{k, j-1}-2 \varphi_{k, j}\right) \varphi_{\ell, j}=\sum_{j=1}^{N}\left(\varphi_{k, j+1}+\varphi_{k, j-1}\right) \varphi_{\ell, j} .
$$

Therefore

$$
\sum_{j=1}^{N} \varphi_{k, j+1} \varphi_{\ell, j}=-\sum_{j=1}^{N} \varphi_{k, j-1} \varphi_{\ell, j}=-\sum_{j=1}^{N} \varphi_{k, j} \varphi_{\ell, j+1}
$$

In other words

$$
\sum_{j=1}^{N}\left[\varphi_{k, j+1} \varphi_{\ell, j}+\varphi_{k, j} \varphi_{\ell, j+1}\right]=0
$$

which, in view of (2.7) is equivalent to (2.6).

The gap between consecutive eigenvalues plays an important role on the analysis of the boundary observability problem, since eigenvalues that are very close produce time harmonics at $x=L$ that are almost indistinguishible for short intervals of time. For the continuous model we have

$$
\sqrt{\lambda_{k+1}}-\sqrt{\lambda_{k}}=\frac{(k+1) \pi}{L}-\frac{k \pi}{L}=\frac{\pi}{L} .
$$

Thus, the gap, $\frac{\pi}{L}$, is independent of the frequency.

However, as we shall see, the gap between consecutive eigenvalues in the discrete problem decreases at high frequencies and it is of the order of $h$ as $h \rightarrow 0$. 
We have

$$
\begin{aligned}
\sqrt{\lambda_{k+1}(h)}-\sqrt{\lambda_{k}(h)} & =\frac{2}{h}\left[\sin \left(\frac{\pi h(k+1)}{2 L}\right)-\sin \left(\frac{\pi h k}{2 L}\right)\right] \\
& =\frac{2}{h}\left[\sin \left(\frac{\pi h k}{2 L}\right)\left(\cos \left(\frac{\pi h}{2 L}\right)-1\right)+\sin \left(\frac{\pi h}{2 L}\right) \cos \left(\frac{\pi h k}{2 L}\right)\right] \\
& \leq \frac{2}{h}\left|1-\cos \left(\frac{\pi h}{2 L}\right)\right|+\frac{\pi}{L} \cos \left(\frac{\pi h k}{2 L}\right)
\end{aligned}
$$

and, taking into account that $(N+1) h=L$,

$$
\begin{aligned}
\cos \left(\frac{\pi h k}{2 L}\right) & =\cos \left(\frac{\pi}{2}+\frac{(k-(N+1)) h \pi}{2 L}\right) \\
& =\sin \left(\frac{((N+1)-k) h \pi}{2 L}\right)
\end{aligned}
$$

and that

$$
\frac{2}{h}\left|1-\cos \left(\frac{\pi h}{2 L}\right)\right| \leq \frac{\pi^{2} h}{2 L^{2}}
$$

we deduce

$$
\sqrt{\lambda_{k+1}(h)}-\sqrt{\lambda_{k}(h)} \leq \frac{\pi^{2} h}{2 L^{2}}+\frac{\pi}{L} \sin \left(\frac{((N+1)-k) h \pi}{2 L}\right) .
$$

Therefore, as soon as

$$
N+1-k \leq j \Leftrightarrow k \geq N+1-j
$$

for some $j \in N$ we have

$$
\sqrt{\lambda_{k+1}(h)}-\sqrt{\lambda_{k}(h)} \leq \frac{\pi^{2} h}{2 L^{2}}+\frac{\pi^{2} j h}{2 L^{2}}=\frac{\pi^{2}}{2 L^{2}} h(j+1) .
$$

This shows that the gap between the roots of the largest $j$ eigenvalues corresponding to the indexes $k=$ $N+1-j, N+2-j, \cdots, N$ is of the order of $h$, with a multiplicative factor that increases as $j$ increases.

In particular, the gap between the largest eigenvalues may be bounded above as follows

$$
\sqrt{\lambda_{N}(h)}-\sqrt{\lambda_{N-1}(h)} \leq \frac{3 \pi^{2}}{2 L^{2}} h \rightarrow 0, \text { as } h \rightarrow 0
$$

Reciprocally, it can be shown that the gap remains bounded below for the low eigenvalues. Indeed,

$$
\sqrt{\lambda_{k+1}(h)}-\sqrt{\lambda_{k}(h)} \geq \frac{2}{h} \sin \left(\frac{\pi h}{2 L}\right) \cos \left(\frac{\pi h k}{2 L}\right)-\frac{\pi^{2} h}{2 L^{2}}=\left[\frac{\pi}{L}-O(h)\right] \cos \left(\frac{\pi h k}{2 L}\right)-\frac{\pi^{2} h}{2 L^{2}} .
$$

The right hand side of this inequality converges to $\frac{\pi}{L}$ as $h \rightarrow 0$ when $k$ remains bounded, or even if $k$ is unbounded but $h k$ is bounded above by $\delta L$ with $0<\delta<1$. Recall that $\pi / L$ is the gap between the roots of the eigenvalues in the continuous model. 


\subsection{Boundary observability of eigenvectors}

The goal of this section is to prove the identity (1.16) of Lemma 1.1.

In view of the explicit values of the eigenvalues and eigenvectors in (2.2) and (2.3) this identity can be checked easily. However, we shall prove it using multiplier techniques.

First of all, we normalize the eigenvector so that

$$
h \sum_{j=1}^{N}\left|\varphi_{j}\right|^{2}=1
$$

Now, (2.5) becomes

$$
h \sum_{j=0}^{N}\left|\frac{\varphi_{j}-\varphi_{j+1}}{h}\right|^{2}=\lambda
$$

Thus,

and so,

$$
\lambda=\frac{2}{h} \sum_{j=1}^{N}\left|\varphi_{j}\right|^{2}-\frac{2}{h} \sum_{j=1}^{N} \varphi_{j} \varphi_{j+1}
$$

$$
\sum_{j=1}^{N} \varphi_{j} \varphi_{j+1}=\frac{1}{h}-\frac{\lambda h}{2}
$$

We multiply in (2.1) by $j\left(\varphi_{j+1}-\varphi_{j-1}\right) / 2$ and add for $j=1, \cdots, N$ (note that this is a discrete version of the multiplier $\left.x \varphi_{x}\right)$. We obtain on the left hand side

$$
\begin{aligned}
-\frac{1}{h^{2}} \sum_{j=1}^{N}\left[\varphi_{j+1}+\varphi_{j-1}-2 \varphi_{j}\right] j \frac{\left(\varphi_{j+1}-\varphi_{j-1}\right)}{2} & =-\frac{1}{h^{2}} \sum_{j=1}^{N} \frac{j}{2}\left[\left|\varphi_{j+1}\right|^{2}-\left|\varphi_{j-1}\right|^{2}\right]+\frac{1}{h^{2}} \sum_{j=1}^{N} j\left(\varphi_{j+1}-\varphi_{j-1}\right) \varphi_{j} \\
& =\frac{1}{h^{2}} \sum_{j=1}^{N}\left|\varphi_{j}\right|^{2}-\frac{N+1}{2 h^{2}}\left|\varphi_{N}\right|^{2}-\frac{1}{h^{2}} \sum_{j=1}^{N} \varphi_{j} \varphi_{j+1} \\
& =\frac{\lambda}{2 h}-\frac{N+1}{2}\left|\frac{\varphi_{N}}{h}\right|^{2}
\end{aligned}
$$

by virtue of (2.14) and (2.16)

On the right hand side we have,

$$
\lambda \sum_{j=1}^{N} j \varphi_{j}\left(\frac{\varphi_{j+1}-\varphi_{j-1}}{2}\right)=-\frac{\lambda}{2} \sum_{j=1}^{N} \varphi_{j} \varphi_{j+1}=-\frac{\lambda}{2}\left(\frac{1}{h}-\frac{\lambda h}{2}\right) .
$$

Therefore

$$
\frac{\lambda}{2 h}-\frac{N+1}{2}\left|\frac{\varphi_{N}}{h}\right|^{2}=-\frac{\lambda}{2}\left(\frac{1}{h}-\frac{\lambda h}{2}\right)
$$

In other words,

$$
\frac{L}{2}\left|\frac{\varphi_{N}}{h}\right|^{2}=\frac{(N+1) h}{2}\left|\frac{\varphi_{N}}{h}\right|^{2}=\frac{\lambda}{2}+\frac{\lambda}{2}-\frac{\lambda^{2} h^{2}}{4}=\lambda\left(1-\frac{\lambda h^{2}}{4}\right) .
$$


Combining (2.18) and (2.15) we deduce that

$$
h \sum_{j=0}^{N}\left|\frac{\varphi_{j}-\varphi_{j+1}}{h}\right|^{2}=\frac{2 L}{\left(4-\lambda h^{2}\right)}\left|\frac{\varphi_{N}}{h}\right|^{2} .
$$

This completes the proof of Lemma 1.1.

Remark 2.1. In Remark 1.1 we have justified the choice of $-u_{N}(t) / h$ as an approximation of $u_{x}(L, t)$. In view of the explicit form of the eigenvectors $\varphi^{k}$ in $(2.3)$ it is immediate to see that for any $k \geq 1$ fixed

$$
-\left.\frac{\varphi_{k, N}}{h} \rightarrow \partial_{x}\left(\sin \left(\frac{k \pi x}{L}\right)\right)\right|_{x=L} \text { as } h \rightarrow 0
$$

which is the normal derivative of the $k$ th eigenfunction $\varphi^{k}(x)=\sin \left(\frac{k \pi x}{L}\right)$ of the continuous wave equation.

For fixed $k \geq 1$, we can also pass to the limit in (1.16) of Lemma 1.1. We then obtain the identity

$$
\int_{0}^{L}\left|\varphi_{x}^{k}(x)\right|^{2} d x=\frac{L}{2}\left|\varphi_{x}^{k}(L)\right|^{2}
$$

Observe however that identity (1.16) degenerates when $\lambda h^{2} \rightarrow 4$ as $h \rightarrow 0$. This is also a typical fact in the numerical approximation of wave equations which is due to the spurious high frequency oscillations that the numerical scheme introduces (see [3]).

\subsection{Proof of the non-uniform observability}

This section is devoted to prove Theorem 1.1. As indicated in the Introduction, it is an immediate consequence of Lemma 1.1. Indeed, let $u$ be the solution of (1.6) associated to the $N$ th eigenvector, i.e.,

$$
u=e^{i \sqrt{\lambda_{N}(h)} t} \varphi^{N} .
$$

According to Lemma 1.1 we have

$$
\int_{0}^{T}\left|\frac{u_{N}(t)}{h}\right|^{2} d t=T\left|\frac{\varphi_{N, N}}{h}\right|^{2}=\frac{T h}{2 L}\left(4-\lambda_{N}(h) h^{2}\right) \sum_{j=0}^{N}\left|\frac{\varphi_{N, j}-\varphi_{N, j+1}}{h}\right|^{2} .
$$

On the other hand

$$
\begin{aligned}
E_{h}(0) & =\frac{h}{2} \sum_{j=0}^{N}\left[\frac{\left|\varphi_{N, j}-\varphi_{N, j+1}\right|^{2}}{h^{2}}+\lambda_{N}(h)\left|\varphi_{N, j}\right|^{2}\right] \\
& =h \sum_{j=0}^{N}\left[\frac{\left|\varphi_{N, j}-\varphi_{N, j+1}\right|^{2}}{h^{2}}\right] .
\end{aligned}
$$

In view of (2.20) and (2.21) we deduce that

$$
\frac{E_{h}(0)}{\int_{0}^{T}\left|u_{N}(t) / h\right|^{2} d t}=\frac{2 L}{T\left(4-\lambda_{N}(h) h^{2}\right)}
$$


Moreover, in view of $(2.2)$ :

$$
\begin{aligned}
\lambda_{N}(h) h^{2} & =4 \sin ^{2}\left(\frac{\pi N h}{2 L}\right)=4 \sin ^{2}\left(\frac{\pi}{2}-\frac{h \pi}{2 L}\right) \\
& =4 \cos ^{2}\left(\frac{h \pi}{2 L}\right) \rightarrow 4 \text { as } h \rightarrow 0 .
\end{aligned}
$$

Combining (2.22) and (2.23), Theorem 1.1 follows.

\subsection{Boundary observability of the discrete wave equation: the multiplier method}

This section is devoted to prove Theorem 1.2 using the multiplier method. As we shall see in Section 2.5 below, the results of this section can be obtained more directly by means of Fourier series techniques. However, we think that the development we present here of the discrete multiplier techniques is of independent interest in view of its potential applications in the analysis of similar problems in several space dimensions.

First of all we establish some basic identities.

Lemma 2.2. (Conservation of energy) For any $h>0$ and $u$ solution of (1.6) we have

$$
E_{h}(t)=E_{h}(0), \quad \forall t \in[0, T] .
$$

Proof. We multiply in (1.6) by $u_{j}^{\prime}(t)$ and add for $j=1, \cdots, N$.

We have

$$
\sum_{j=1}^{N} u_{j}^{\prime \prime} u_{j}^{\prime}=\frac{1}{h^{2}} \sum_{j=1}^{N}\left(u_{j+1}+u_{j-1}-2 u_{j}\right) u_{j}^{\prime}
$$

On the other hand,

$$
\sum_{j=1}^{N} u_{j}^{\prime \prime} u_{j}^{\prime}=\frac{1}{2} \frac{d}{d t} \sum_{j=1}^{N}\left|u_{j}^{\prime}\right|^{2}
$$

and

$$
-\sum_{j=1}^{N}\left(u_{j+1}+u_{j-1}-2 u_{j}\right) u_{j}^{\prime}=\frac{1}{2} \frac{d}{d t} \sum_{j=0}^{N}\left|u_{j}-u_{j+1}\right|^{2} .
$$

Combining (2.25-2.27) we deduce that

$$
\frac{d}{d t} \sum_{j=0}^{N}\left[\left|u_{j}^{\prime}\right|^{2}+\left|\frac{u_{j}-u_{j+1}}{h}\right|^{2}\right]=0
$$

which is equivalent to $(2.24)$.

Lemma 2.3. For any $h>0$ and $u$ solution of (1.6) we have

$$
\frac{h}{2} \sum_{j=0}^{N} \int_{0}^{T}\left[u_{j}^{\prime} u_{j+1}^{\prime}+\left|\frac{u_{j+1}-u_{j}}{h}\right|^{2}\right] d t+\left.X_{h}(t)\right|_{0} ^{T}=\frac{L}{2} \int_{0}^{T}\left|\frac{u_{N}(t)}{h}\right|^{2} d t
$$


with

$$
X_{h}(t)=h \sum_{j=1}^{N} j\left(\frac{u_{j+1}-u_{j-1}}{2}\right) u_{j}^{\prime}
$$

Remark 2.2. Identity (2.28) is the discrete analogue of the well-known identity for the $1-d$ wave equation (1.1) obtained by multipliers that reads as follows (see [11]):

$$
\frac{1}{2} \int_{0}^{T} \int_{0}^{L}\left[\left|u_{t}\right|^{2}+\left|u_{x}\right|^{2}\right] d x d t+\left.X(t)\right|_{0} ^{T}=\frac{L}{2} \int_{0}^{T}\left|u_{x}(1, t)\right|^{2} d t
$$

with

$$
X(t)=\int_{0}^{L} x u_{x} u_{t} d x
$$

Note that the main difference between (2.28)-(2.29) and (2.30)-(2.31) is that, in (2.28), we get

$$
h \sum_{j=0}^{N} \int_{0}^{T} u_{j}^{\prime} u_{j+1}^{\prime} d t
$$

as a discretization of $\int_{0}^{T} \int_{0}^{L} u_{t}^{2} d x d t$, which is not a positive definite quantity.

Proof of Lemma 2.3. We multiply in (1.6) by $j\left(u_{j+1}-u_{j-1}\right) / 2$ which is a discrete version of the classical multiplier $x u_{x}$ for the wave equation. We obtain

$$
\sum_{j=1}^{N} \int_{0}^{T} u_{j}^{\prime \prime} j \frac{\left(u_{j+1}-u_{j-1}\right)}{2} d t=\frac{1}{h^{2}} \sum_{j=1}^{N} \int_{0}^{T}\left(u_{j+1}+u_{j-1}-2 u_{j}\right) j \frac{\left(u_{j+1}-u_{j-1}\right)}{2} d t .
$$

We now develop the two terms in this identity. For the first one we have:

$$
\begin{aligned}
\sum_{j=1}^{N} \int_{0}^{T} u_{j}^{\prime \prime} j \frac{\left(u_{j+1}-u_{j-1}\right)}{2} d t & =-\sum_{j=1}^{N} \int_{0}^{T} u_{j}^{\prime} j \frac{\left(u_{j+1}^{\prime}-u_{j-1}^{\prime}\right)}{2} d t+\left.\sum_{j=1}^{N} u_{j}^{\prime} j \frac{\left(u_{j+1}-u_{j-1}\right)}{2}\right|_{0} ^{T} \\
& =\frac{1}{2} \sum_{j=1}^{N} \int_{0}^{T} u_{j}^{\prime} u_{j+1}^{\prime} d t+\left.\sum_{j=1}^{N} u_{j}^{\prime} j \frac{\left(u_{j+1}-u_{j-1}\right)}{2}\right|_{0} ^{T}
\end{aligned}
$$


On the other hand,

$$
\begin{aligned}
\sum_{j=1}^{N} \int_{0}^{T}\left(u_{j+1}+u_{j-1}-2 u_{j}\right) j \frac{\left(u_{j+1}-u_{j-1}\right)}{2} d t & =\frac{1}{2} \sum_{j=1}^{N} \int_{0}^{T} j\left[\left|u_{j+1}\right|^{2}-\left|u_{j-1}\right|^{2}\right] d t \\
& -\sum_{j=1}^{N} \int_{0}^{T} j u_{j}\left(u_{j+1}-u_{j-1}\right) d t \\
& =-\sum_{j=1}^{N} \int_{0}^{T}\left|u_{j}\right|^{2}+\frac{(N+1)}{2} \int_{0}^{T}\left|u_{N}\right|^{2} d t+\sum_{j=1}^{N} \int_{0}^{T} u_{j} u_{j+1} d t \\
& =-\frac{1}{2} \sum_{j=0}^{N} \int_{0}^{T}\left|u_{j}-u_{j+1}\right|^{2} d t+\frac{(N+1)}{2} \int_{0}^{T}\left|u_{N}\right|^{2} d t .(2.34)
\end{aligned}
$$

Combining (2.32-2.34) we deduce that

$$
\begin{aligned}
\frac{h}{2} \sum_{j=0}^{N} \int_{0}^{T}\left[u_{j}^{\prime} u_{j+1}^{\prime}+\frac{\left|u_{j}-u_{j+1}\right|^{2}}{h^{2}}\right] d t+\left.X_{h}(t)\right|_{0} ^{T} & =\frac{(N+1) h}{2} \int_{0}^{T}\left|\frac{u_{N}(t)}{h}\right|^{2} d t \\
& =\frac{L}{2} \int_{0}^{T}\left|\frac{u_{N}(t)}{h}\right|^{2} d t
\end{aligned}
$$

This concludes the proof of the Lemma.

Lemma 2.4. (Equipartition of energy) For any $h>0$ and $u$ solution of (1.6) the following identity holds:

$$
-h \sum_{j=1}^{N} \int_{0}^{T}\left|u_{j}^{\prime}\right|^{2} d t+h \sum_{j=0}^{N} \int_{0}^{T}\left|\frac{u_{j}-u_{j+1}}{h}\right|^{2} d t+\left.Y_{h}(t)\right|_{0} ^{T}=0
$$

with

$$
Y_{h}(t)=\left.h \sum_{j=1}^{N} u_{j}^{\prime} u_{j}\right|_{0} ^{T}
$$

Proof. We multiply in (1.6) by $u_{j}$. Note that this is the discrete version of the classical multiplier $u$ for the wave equation. We obtain

$$
\sum_{j=1}^{N} \int_{0}^{T} u_{j}^{\prime \prime} u_{j} d t-\sum_{j=1}^{N} \int_{0}^{T} \frac{\left(u_{j+1}+u_{j-1}-2 u_{j}\right)}{h^{2}} u_{j} d t=0
$$

On the other hand,

$$
\sum_{j=1}^{N} \int_{0}^{T} u_{j}^{\prime \prime} u_{j} d t=-\sum_{j=1}^{N} \int_{0}^{T}\left|u_{j}^{\prime}\right|^{2} d t+\left.\sum_{j=1}^{N} u_{j}^{\prime} u_{j}\right|_{0} ^{T}
$$


and, again,

$$
\sum_{j=1}^{N}\left(u_{j+1}+u_{j-1}-2 u_{j}\right) u_{j}=-\sum_{j=0}^{N}\left|u_{j}-u_{j+1}\right|^{2}
$$

Combining (2.37-2.39) we deduce that (2.35) holds.

We may now proceed to the proof of Theorem 1.2 .

In view of the conservation of energy, identity (2.28) may be rewritten as

$$
T E_{h}(0)+\frac{h}{2} \sum_{j=0}^{N} \int_{0}^{T}\left[u_{j}^{\prime} u_{j+1}^{\prime}-\left|u_{j}^{\prime}\right|^{2}\right] d t+\left.X_{h}(t)\right|_{0} ^{T}=\frac{L}{2} \int_{0}^{T}\left|\frac{u_{N}(t)}{h}\right|^{2} d t .
$$

On the other hand

$$
\sum_{j=0}^{N} \int_{0}^{T}\left[u_{j}^{\prime} u_{j+1}^{\prime}-\left|u_{j}^{\prime}\right|^{2}\right] d t=-\frac{1}{2} \sum_{j=0}^{N} \int_{0}^{T}\left|u_{j}^{\prime}-u_{j+1}^{\prime}\right|^{2} d t
$$

The right hand side of (2.41) can be estimated as follows. Let $\Lambda$ be the largest eigenvalue in the Fourier development of $u$. Then

$$
u=\sum_{\left|\mu_{k}\right| \leq \sqrt{\Lambda}} a_{k} e^{i \mu_{k} t} \varphi^{k}
$$

with $\mu_{k}=\sqrt{\lambda_{k}}$ for $k>0$ and $\mu_{-k}=-\mu_{k}$. Therefore

$$
u^{\prime}=i \sum_{\left|\mu_{k}\right| \leq \sqrt{\Lambda}} a_{k} \mu_{k} e^{i \mu_{k} t} \varphi^{k} .
$$

Thus

$$
\begin{aligned}
& \sum_{j=0}^{N}\left|u_{j}^{\prime}-u_{j+1}^{\prime}\right|^{2}=\sum_{j=0}^{N}\left|\sum_{\left|\mu_{k}\right| \leq \sqrt{\Lambda}} a_{k} \mu_{k} e^{i \mu_{k} t}\left(\varphi_{k, j}-\varphi_{k, j+1}\right)\right|^{2} \\
& =\sum_{j=0}^{N} \sum_{\left|\mu_{k}\right| \leq \sqrt{\Lambda}} \mu_{k}^{2}\left|a_{k}\right|^{2}\left|\varphi_{k, j}-\varphi_{k, j+1}\right|^{2} \\
& +\sum_{j=0}^{N} \sum_{\substack{\left|\mu_{k}\right| \leq \sqrt{\Lambda} \\
\left|\mu_{\ell}\right| \leq \sqrt{\Lambda} \\
\mu_{k} \neq \mu_{\ell}}} \mu_{k} \mu_{\ell} e^{i\left(\mu_{k}-\mu_{t}\right) t}\left(\varphi_{k, j}-\varphi_{k, j+1}\right)\left(\varphi_{\ell, j}-\varphi_{\ell, j+1}\right) .
\end{aligned}
$$


In view of the identities $(2.5-2.6)$ the term in $(2.44)$ can be rewritten as

$$
\begin{aligned}
\sum_{j=0}^{N}\left|u_{j}^{\prime}-u_{j+1}^{\prime}\right|^{2} & =\sum_{\left|\mu_{k}\right| \leq \sqrt{\Lambda}}\left|a_{k}\right|^{2} \lambda_{k}^{2} h^{2} \sum_{j=1}^{N}\left|\varphi_{k, j}\right|^{2} \\
& \leq \Lambda \sum_{\left|\mu_{k}\right| \leq \sqrt{\Lambda}}\left|a_{k}\right|^{2} \lambda_{k} h^{2} \sum_{j=1}^{N}\left|\varphi_{k, j}\right|^{2} \\
& =\Lambda h^{2} \sum_{j=1}^{N}\left|u_{j}^{\prime}\right|^{2}
\end{aligned}
$$

Therefore

$$
\sum_{j=0}^{N} \int_{0}^{T}\left[u_{j}^{\prime} u_{j+1}^{\prime}-\left|u_{j}^{\prime}\right|^{2}\right] d t \geq-\frac{\Lambda h^{2}}{2} \sum_{j=1}^{N} \int_{0}^{T}\left|u_{j}^{\prime}\right|^{2} d t .
$$

Combining (2.40) and (2.45) we deduce that

$$
T E_{h}(0)-\frac{\Lambda h^{2}}{4} h \sum_{j=0}^{N} \int_{0}^{T}\left|u_{j}^{\prime}\right|^{2} d t+\left.X_{h}(t)\right|_{0} ^{T} \leq \frac{L}{2} \int_{0}^{T}\left|\frac{u_{N}(t)}{h}\right|^{2} d t .
$$

In view of the equipartition of energy identity (2.35) it follows that

$$
h \sum_{j=1}^{N} \int_{0}^{T}\left|u_{j}^{\prime}\right|^{2} d t=\int_{0}^{T} E_{h}(t) d t+\left.\frac{1}{2} Y_{h}(t)\right|_{0} ^{T}=T E_{h}(0)+\left.\frac{1}{2} Y_{h}(t)\right|_{0} ^{T} .
$$

Combining (2.46) and (2.47) we deduce that

$$
T\left(1-\frac{\Lambda h^{2}}{4}\right) E_{h}(0)+\left.Z_{h}(t)\right|_{0} ^{T} \leq \frac{L}{2} \int_{0}^{T}\left|\frac{u_{N}(t)}{h}\right|^{2} d t
$$

with

$$
\begin{aligned}
Z_{h}(t) & =X_{h}(t)-\frac{\Lambda h^{2}}{8} Y_{h}(t) \\
& =h \sum_{j=1}^{N} u_{j}^{\prime}\left[j \frac{\left(u_{j+1}-u_{j-1}\right)}{2}-\frac{\Lambda h^{2}}{8} u_{j}\right],
\end{aligned}
$$

for every solution of (1.6) in which $\Lambda$ is the largest eigenvalue entering in its Fourier expansion.

The following provides an estimate on the term $Z_{h}$ :

Lemma 2.5. For any $h>0, t \in[0, T]$ and $u$ solution of (1.6) in which $\Lambda$ is the upper bound on the eigenvalues entering in its Fourier development, it follows that

$$
\left|Z_{h}(t)\right| \leq \sqrt{L^{2}-\frac{\Lambda h^{4}}{16}+\frac{3 \Lambda h^{2}}{16 \lambda_{1}}} E_{h}(0) .
$$


Proof. We do not make explicit the time dependence to simplify the notation. We have

$$
\left|Z_{h}\right| \leq h\left[\sum_{j=1}^{N}\left|u_{j}^{\prime}\right|^{2}\right]^{1 / 2}\left[\sum_{j=1}^{N}\left|j \frac{\left(u_{j+1}-u_{j-1}\right)}{2}+\eta u_{j}\right|^{2}\right]^{1 / 2}
$$

with $\eta=-\Lambda h^{2} / 8$.

On the other hand

$$
\begin{aligned}
h \sum_{j=1}^{N}\left|\frac{j\left(u_{j+1}-u_{j-1}\right)}{2}+\eta u_{j}\right|^{2} & =h \sum_{j=1}^{N}\left[\frac{j^{2}}{4}\left|u_{j+1}-u_{j-1}\right|^{2}+\eta^{2} u_{j}^{2}+\eta j\left(u_{j+1}-u_{j-1}\right) u_{j}\right] \\
& \leq h \sum_{j=0}^{N}\left[\frac{j^{2}}{2}\left|u_{j+1}-u_{j}\right|^{2}+\frac{j^{2}}{2}\left|u_{j}-u_{j-1}\right|^{2}+\eta^{2} u_{j}^{2}-\eta u_{j} u_{j+1}\right] \\
& \leq L^{2} h \sum_{j=0}^{N} \frac{\left|u_{j}-u_{j+1}\right|^{2}}{h^{2}}-|\eta| h \sum_{j=1}^{N}\left(u_{j}^{2}-u_{j} u_{j+1}\right)+\left[\eta^{2}+|\eta|\right] h \sum_{j=1}^{N} u_{j}^{2} \\
& =\left(L^{2}-\frac{|\eta| h^{2}}{2}\right) h \sum_{j=0}^{N}\left|\frac{u_{j}-u_{j+1}}{h}\right|^{2}+\left[\eta^{2}+|\eta|\right] h \sum_{j=1}^{N} u_{j}^{2} \\
& =\left[L^{2}-\frac{|\eta| h^{2}}{2}+\frac{\left(\eta^{2}+|\eta|\right)}{\lambda_{1}}\right] h \sum_{j=0}^{N}\left|\frac{u_{j}-u_{j+1}}{h}\right|^{2} \\
& \leq\left[L^{2}-\frac{\Lambda h^{4}}{16}+\frac{3 \Lambda h^{2}}{16 \lambda_{1}}\right] h \sum_{j=0}^{N}\left|\frac{u_{j}-u_{j+1}}{h}\right|^{2}
\end{aligned}
$$

In the last step we have used the fact that

$$
h \sum_{j=1}^{N} u_{j}^{2} \leq \frac{1}{\lambda_{1}} h \sum_{j=0}^{N}\left|\frac{u_{j}-u_{j+1}}{h}\right|^{2}
$$

which is the discrete version of Poincaré's inequality and can be deduced easily from (2.5-2.6). We have also used that $\eta^{2}+|\eta| \leq \frac{3}{2}|\eta|$ which is a consequence of the fact that $|\eta|=\Lambda h^{2} / 8 \leq 1 / 2$ as it is immediately seen from (2.2).

Combining (2.51-2.52) we deduce that

$$
\begin{aligned}
\left|Z_{h}\right| & \leq \sqrt{L^{2}-\frac{\Lambda h^{4}}{16}+\frac{3 \Lambda h^{2}}{16 \lambda_{1}}}\left(h \sum_{j=1}^{N}\left|u_{j}^{\prime}\right|^{2}\right)^{1 / 2}\left(h \sum_{j=0}^{N}\left|\frac{u_{j+1}-u_{j}}{h}\right|^{2}\right)^{1 / 2} \\
& \leq \sqrt{L^{2}-\frac{\Lambda h^{4}}{16}+\frac{3 \Lambda h^{2}}{16 \lambda_{1}}} E_{h}(0)
\end{aligned}
$$

Using (2.48) and (2.50) we deduce that

$$
\left[T\left(1-\Lambda h^{2} / 4\right)-2 \sqrt{L^{2}-\frac{\Lambda h^{4}}{16}+\frac{3 \Lambda h^{2}}{16 \lambda_{1}}}\right] E_{h}(0) \leq \frac{L}{2} \int_{0}^{T}\left|\frac{u_{N}(t)}{h}\right|^{2} d t .
$$


As a consequence of (2.54) and taking into account that $\Lambda=\gamma / h^{2}$ in the class of solutions $\mathcal{C}_{h}(\gamma)$ of system $(1.6)$ we deduce that

$$
E_{h}(0) \leq \frac{L}{2\left(T(1-\gamma / 4)-2 \sqrt{L^{2}+\gamma\left(3 / \lambda_{1}-h^{2}\right) / 16}\right)} \int_{0}^{T}\left|\frac{u_{N}(t)}{h}\right|^{2} d t
$$

provided

$$
T>2 \sqrt{L^{2}-\frac{\gamma h^{2}}{16}+\frac{3 \gamma}{16 \lambda_{1}}} /(1-\gamma / 4)
$$

Taking into account that $\lambda_{1} \geq \pi^{2} / 2 L^{2}$ for $h$ sufficiently small, the statement of Theorem 1.2 holds with

$$
T(\gamma)=\frac{2 \sqrt{L^{2}\left(1+3 \gamma / 8 \pi^{2}\right)-\gamma h^{2} / 16}}{1-\gamma / 4}
$$

and

$$
C(T, \gamma)=\frac{L}{2\left(T(1-\gamma / 4)-2 \sqrt{L^{2}\left(1+3 \gamma / 8 \pi^{2}\right)-\gamma h^{2} / 16}\right)} .
$$

\subsection{Boundary observability of the semi-discrete wave equation: non-harmonic Fourier series}

In this section we prove Theorem 1.2 using well known results from the theory of nonharmonic Fourier series.

To do that we need an estimate between the roots of consecutive eigenvalues entering in the Fourier development of the solutions of (1.6) in the class $\mathcal{C}_{h}(\gamma)$. We have

Lemma 2.6. Assume that

$$
\gamma=4 \sin ^{2}\left(\frac{\pi \varepsilon}{2}\right)
$$

for some $0 \leq \varepsilon<1$. Then

$$
\sqrt{\lambda_{j+1}(h)}-\sqrt{\lambda_{j}(h)} \geq \frac{\pi}{L} \cos \left(\frac{\pi \varepsilon}{2}\right)
$$

for all eigenvalues in the range

$$
\lambda h^{2} \leq \gamma
$$

Remark 2.3. Note that every $0 \leq \gamma<4$ can be written in the form (2.57) for some $0 \leq \varepsilon<1$. Note also that the gap given in (2.58) tends to $\pi / L$, the gap in the continuous wave equation, as $\varepsilon \rightarrow 0$, i.e., as $\gamma \rightarrow 0$. This is consistent with the estimates of Section 2.2 in which we observed that the gap for large eigenvalues is of the order of $h$.

Proof of Lemma 2.6. In view of the expression (2.2) the eigenvalues $\lambda$ satisfy (2.59) with $\gamma$ as in (2.57) if and only if

$$
(j+1) h \leq \varepsilon L
$$

Let us now compute the gap:

$$
\sqrt{\lambda_{j+1}(h)}-\sqrt{\lambda_{j}(h)}=\frac{2}{h}\left[\sin \left(\frac{\pi(j+1) h}{2 L}\right)-\sin \left(\frac{\pi j h}{2 L}\right)\right]=\frac{\pi}{L} \cos (\xi)
$$


for some $\xi \in\left[\frac{\pi j h}{2 L}, \frac{\pi(j+1) h}{2 L}\right]$. In view of (2.60), $0 \leq \xi \leq \frac{\pi \varepsilon}{2}$ and therefore $\cos \xi \geq \cos (\pi \varepsilon / 2)$. Thus (2.58) holds.

According to Ingham's inequality [7] and in view of Lemma 2.6 it follows that for any $0<\varepsilon<1$ and $T>\frac{2 L}{\cos (\pi \varepsilon / 2)}$ there exist positive constants $C_{1}(T, \varepsilon), C_{2}(T, \varepsilon)>0$ such that

$$
\begin{aligned}
C_{1}(T, \varepsilon) \sum_{\left|\mu_{k}\right| h \leq \sqrt{\gamma(\varepsilon)}}\left|a_{k}\right|^{2} & \leq \int_{0}^{T}\left|\sum_{\left|\mu_{k}\right| h \leq \sqrt{\gamma(\varepsilon)}} a_{k} e^{i \mu_{k} t}\right|^{2} d t \\
& \leq C_{2}(T, \varepsilon) \sum_{\lambda h^{2} \leq \gamma(\varepsilon)}\left|a_{k}\right|^{2}
\end{aligned}
$$

with

$$
\gamma(\varepsilon)=4 \sin ^{2}(\pi \varepsilon / 2)
$$

On the other hand, in the range of eigenvalues

$$
\lambda h^{2} \leq \gamma(\varepsilon)
$$

according to the identity of Lemma 1.1 it follows that

$$
h \sum_{j=0}^{N}\left|\frac{\varphi_{j+1}-\varphi_{j}}{h}\right|^{2} \leq \frac{2 L}{4-\gamma(\varepsilon)}\left|\frac{\varphi_{N}}{h}\right|^{2}=\frac{L}{2 \cos ^{2}(\pi \varepsilon / 2)}\left|\frac{\varphi_{N}}{h}\right|^{2}
$$

for any eigenvector $\varphi=\left(\varphi_{1}, \cdots, \varphi_{N}\right)$ associated to an eigenvalue $\lambda$ in the range (2.64).

Let us now consider a solution $u$ of (1.6) in the class $\mathcal{C}_{h}(\gamma(\varepsilon))$. It can be written as

$$
u=\sum_{\left|\mu_{k}(h)\right| h \leq \sqrt{\gamma(\varepsilon)}} a_{k} e^{i \mu_{k}(h) t} \varphi^{k} .
$$

According to (2.62) and (2.65) we deduce that, for $T>2 L / \cos (\pi \varepsilon / 2)$,

$$
\begin{aligned}
\int_{0}^{T}\left|\frac{u_{N}(t)}{h}\right|^{2} d t & =\int_{0}^{T}\left|\sum_{\left|\mu_{k}\right| h \leq \sqrt{\gamma(\varepsilon)}} a_{k} e^{i \mu_{k}(h) t} \varphi_{k, N}\right|^{2} \\
& \geq C_{1}(T, \varepsilon) \sum_{\left|\mu_{k}\right| h \leq \sqrt{\gamma(\varepsilon)}}\left|a_{k}\right|^{2}\left|\varphi_{k, N}\right|^{2} \\
& \geq \frac{C_{1}(T, \varepsilon) L}{2 \cos ^{2}(\pi \varepsilon / 2)} \sum_{\left|\mu_{k}\right| h \leq \sqrt{\gamma(\varepsilon)}}\left|a_{k}\right|^{2} h \sum_{j=0}^{N}\left|\frac{\varphi_{k, j+1}-\varphi_{k, j}}{h}\right|^{2} .
\end{aligned}
$$


Moreover,

$$
\begin{aligned}
E_{h}(0) & =\frac{1}{2} \sum_{\left|\mu_{k}\right| h \leq \sqrt{\gamma(\varepsilon)}}\left[\left|a_{k}\right|^{2} \lambda_{k} h \sum_{j=1}^{N}\left|\varphi_{k, j}\right|^{2}\right]+\frac{1}{2} \sum_{\left|\mu_{k}\right| h \leq \sqrt{\gamma(\varepsilon)}}\left[\left|a_{k}\right|^{2} h \sum_{j=0}^{N}\left|\frac{\varphi_{k, j+1}-\varphi_{k, j}}{h}\right|^{2}\right] \\
& =\sum_{\left|\mu_{k}\right| h \leq \sqrt{\gamma(\varepsilon)}}\left[\left|a_{k}\right|^{2} h \sum_{j=0}^{N}\left|\frac{\varphi_{k, j+1}-\varphi_{k, j}}{h}\right|^{2}\right] .
\end{aligned}
$$

Therefore, as a consequence of (2.67) it follows that

$$
E_{h}(0) \leq \frac{2 \cos ^{2}(\pi \varepsilon / 2)}{L C_{1}(T, \varepsilon)} \int_{0}^{T}\left|\frac{u_{N}(t)}{h}\right|^{2} d t
$$

for any $T>2 L / \cos (\pi \varepsilon / 2)$ and for any $u \in \mathcal{C}_{h}(\gamma(\varepsilon))$.

Therefore, Theorem 1.2 holds with

$$
T(\gamma)=\frac{2 L}{\sqrt{1-\gamma / 4}}
$$

and

$$
C(T, \gamma)=\frac{2(1-\gamma / 4)}{L C_{1}(T, \varepsilon)}
$$

provided $\gamma=\gamma(\varepsilon)$.

Observe that the estimate (2.70) obtained by Ingham's inequality for the observability time is slightly better than (2.55) obtained by multipliers. However both expressions coincide in the limit when $h \rightarrow 0$.

Note also that, according to (2.62), the reverse inequality also holds, i.e.,

$$
\int_{0}^{T}\left|\frac{u_{N}(t)}{h}\right|^{2} d t \leq C E_{h}(0)
$$

However, from the identity (2.28) it is easy to see that (2.72) holds for all $h>0$, every solution $u$ of (1.6) and all $T>0$ with $C=C(T)>0$ independent of $h$.

\section{Finite-ELEMENT SEMI-DisCRETIZATION}

\subsection{Problem formulation}

Let us consider the finite-element space semi-discretization of the wave equation (1.1):

$$
\left\{\begin{array}{l}
\frac{2}{3} u_{j}^{\prime \prime}+\frac{1}{6} u_{j+1}^{\prime \prime}+\frac{1}{6} u_{j-1}^{\prime \prime}=\frac{\left[u_{j+1}+u_{j-1}-2 u_{j}\right]}{h^{2}}, 0<t<T, j=1, \cdots, N \\
u_{0}=u_{N+1}=0 .
\end{array}\right.
$$

Let us recall that system (3.1) is obtained by a Galerkin approximation of the wave equation when the basis of $H_{0}^{1}(0, L)$ is built by means of finite elements

$$
e_{j}(x)=\left[1-\frac{\left|x-x_{j}\right|}{h}\right]^{+}, j=1, \cdots, N
$$

with $x_{j}=j h$. 
The Galerkin approximation of (1.1) is given by

$$
\left\{\begin{array}{l}
u=\sum_{j=1}^{N} u_{j}(t) e_{j}(x) \\
\int_{0}^{L} u^{\prime \prime} e_{k} d x=\int_{0}^{L} u_{x} e_{k, x} d x, \quad 0<t<T, \quad \forall k=1, \cdots, N .
\end{array}\right.
$$

System (3.1) can be easily derived taking into account that

$$
\begin{aligned}
& \left\{\begin{array}{l}
\int_{0}^{1} e_{j} e_{k} d x=\frac{h}{6} \text { if } j=j+1, j-1, \int_{0}^{L} e_{j} e_{k} d x=0 \text { if }|k-j| \geq 2 \\
\int_{0}^{L}\left|e_{j}\right|^{2} d x=\frac{2 h}{3}, \forall j=1, \cdots, N,
\end{array}\right. \\
& \left\{\begin{array}{l}
\int_{0}^{L} e_{j, x} e_{k, x} d x=-\frac{1}{h} \text { if } k=j-1, j+1 ; \int_{0}^{T} e_{j, x} e_{k, x} d x=0 \text { if }|k-j| \geq 2, \\
\int_{0}^{L}\left|e_{j, x}\right|^{2} d x=\frac{2}{h} .
\end{array}\right.
\end{aligned}
$$

The conserved energy for system (3.1) is given by

$$
E_{h}(t)=\frac{h}{6} \sum_{j=1}^{N}\left|u_{j}^{\prime}\right|^{2}+\frac{h}{12} \sum_{j=0}^{N}\left|u_{j}^{\prime}+u_{j+1}^{\prime}\right|^{2}+\frac{h}{2} \sum_{j=0}^{N}\left|\frac{u_{j+1}-u_{j}}{h}\right|^{2},
$$

i.e.,

$$
E_{h}(t)=E_{h}(0), \quad \forall 0<t<T
$$

for all $h>0$ and for every solution of (3.1).

As in Section 2 above, the goal is to obtain observability inequalities of the form

$$
E_{h}(0) \leq C \int_{0}^{T}\left|\frac{u_{N}(t)}{h}\right|^{2} d t
$$

Observe that we adopt $-u_{N}(t) / h$ as approximation of the normal derivative $u_{x}(L, t)$. We refer to Remarks 1.1 and 2.1 above for a detailed discussion of this issue.

Let us see first that the constant $C$ in (3.8) may not be uniform when $h \rightarrow 0$. For that we analyze the eigenvectors of the system:

$$
\left\{\begin{array}{l}
-\left[\frac{\varphi_{j+1}+\varphi_{j-1}-2 \varphi_{j}}{h^{2}}\right]=\lambda\left[\frac{2}{3} \varphi_{j}+\frac{1}{6} \varphi_{j+1}+\frac{1}{6} \varphi_{j-1}\right], j=1, \cdots, N \\
\varphi_{0}=\varphi_{N+1}=0
\end{array}\right.
$$

We denote by $0<\lambda_{1}<\lambda_{2}<\cdots<\lambda_{N}$ the eigenvalues of $(3.9)$ and by $\left\{\varphi^{k}\right\}_{k=1}^{N}$ the associated eigenvectors.

Lemma 3.1. For any $h>0$ and any eigenvector of system (3.9) the following identity holds:

$$
h \sum_{j=0}^{N}\left|\frac{\varphi_{j+1}-\varphi_{j}}{h}\right|^{2}=\frac{6+\lambda h^{2}}{12-\lambda h^{2}} L\left|\frac{\varphi_{N}}{h}\right|^{2}
$$


Moreover, as we shall see $\lambda \leq 12 h^{-2}$ for any $h>0$ and any eigenvalue and

$$
\lambda_{N}(h) h^{2} \rightarrow 12 \text { as } h \rightarrow 0
$$

Therefore, as in the case of the finite-difference it follows that:

Theorem 3.1. For any $T>0$,

$$
u \quad \sup _{\text {solution of }(3.1)}\left[\frac{E_{h}(0)}{\int_{0}^{T}\left|\frac{u_{N}(t)}{h}\right|^{2} d t}\right] \rightarrow \infty
$$

as $h \rightarrow 0$.

In order to prove uniform observability results, for any $0<\gamma<12$ we introduce the class of solutions of (3.1) in which only the terms of the Fourier development corresponding to $\lambda h^{2} \leq \gamma$ do not vanish. More precisely,

$$
\mathcal{C}_{h}(\gamma)=\left\{u \text { solution of }(3.1): u=\sum_{|\mu| h \leq \sqrt{\gamma}} a_{k} e^{i \mu_{k}(h) t} \varphi^{k}\right\}
$$

where, as in the previous section $\mu_{k}(h)=\sqrt{\lambda_{k}(h)}$ when $k \geq 1$ and $\mu_{-k}(h)=-\mu_{k}(h)$.

The following holds:

Theorem 3.2. For any $0<\gamma<12$ there exists $T(\gamma)>0$ such that for any $T>T(\gamma)$ there exists a positive constant $C(T, \gamma)$ such that

$$
E_{h}(0) \leq C(T, \gamma) \int_{0}^{T}\left[\left|\frac{u_{N}(t)}{h}\right|^{2}+\frac{\left|u_{N}^{\prime}(t)\right|^{2}}{6}\right] d t
$$

for any solution $u$ of $(3.1)$ in the class $\mathcal{C}_{h}(\gamma)$. Moreover,

(a) $T(\gamma) \nearrow \infty$ as $\gamma \nearrow 12$ and $T(\gamma) \searrow 2 L$ as $\gamma \searrow 0$

(b) $C(T, \gamma) \rightarrow L /[2(T-2 L)]$ as $\gamma \searrow 0$.

Note that an extra term $\int_{0}^{T}\left|u_{N}^{\prime}(t)\right|^{2} d t$ appears on the right hand side of the observability inequality (3.14). This was not the case in the context of the finite-difference semi-discretization. We shall see that, by using Ingham's inequality, this extra term can be absorved by the term $\int_{0}^{T}\left|u_{N}(t) / h\right|^{2} d t$ by increasing the observability constant.

As in Section 2, the observability inequality (3.14) can be directly proved by Ingham's inequality. However we shall derive it using multipliers.

The rest of this section is organized as follows. First we analyze the spectrum of the system. Then we derive observability identity (3.10) for the eigenvectors. Then we prove Theorem 3.2 by multipliers. Finally, using Ingham's inequality we absorve the term $\int_{0}^{T}\left|u_{N}^{\prime}(t)\right|^{2} d t$ from the right hand side of (3.14).

\subsection{Spectral analysis}

Eigenvalues and eigenvectors of the system (3.9) are those of the matrix $M^{-1} A$, where $A$ is the matrix in Section 2 and $M$ is the matrix

$$
M=\left(\begin{array}{ccccc}
2 / 3 & 1 / 6 & & & \mathbf{0} \\
1 / 6 & 2 / 3 & 1 / 6 & & \\
\cdot & \cdot & \cdot & . & \cdot \\
& & 1 / 6 & 2 / 3 & 1 / 6 \\
\mathbf{0} & & & 1 / 6 & 2 / 3
\end{array}\right) .
$$


In order to compute the spectrum, consider a nonsingular matrix $P$ such that $P^{-1} A P=D, D$ being a diagonal matrix. Taking

$$
J=\left(\begin{array}{ccccc}
0 & 1 & & & \mathbf{0} \\
1 & 0 & 1 & & \\
\cdot & \cdot & \cdot & \cdot & \cdot \\
& & 1 & 0 & 1 \\
\mathbf{0} & & & 1 & 0
\end{array}\right)
$$

we have $A=\frac{1}{h^{2}}(2 I-J)$ and then

$$
P^{-1} J P=2 I-h^{2} D
$$

Now, since $M=\frac{1}{3}\left(2 I+\frac{1}{2} J\right)$, then

$$
P^{-1} M P=I-\frac{h^{2}}{6} D
$$

which is a diagonal matrix. Thus, matrix $M^{-1} A$ and $A$ have the same eigenvectors and, consequently, the eigenfunctions of the system (3.9) are those of the system (2.1). Moreover,

$$
\lambda_{k}\left(M^{-1} A\right)=\frac{\lambda_{k}(A)}{1-\frac{h^{2}}{6} \lambda_{k}(A)}=\frac{6}{h^{2}}\left[\frac{\sin ^{2}\left(\frac{k \pi h}{2 L}\right)}{\frac{3}{2}-\sin ^{2}\left(\frac{k \pi h}{2 L}\right)}\right]=\frac{6}{h^{2}}\left[\frac{1-\cos \left(\frac{k \pi h}{L}\right)}{2+\cos \left(\frac{k \pi h}{L}\right)}\right] .
$$

As we mentioned above

$$
\lambda_{N}(h) h^{2} \rightarrow 12
$$

Indeed,

$$
\begin{aligned}
\lambda_{N}(h) h^{2} & =6\left[\frac{1-\cos (N \pi h / L)}{2+\cos (N \pi h / L)}\right]=6\left[\frac{1-\cos (\pi-h \pi / L)}{2+\cos (\pi-h \pi / L)}\right] \\
& =6\left[\frac{1+\cos (h \pi / L)}{2-\cos (h \pi / L)}\right] \rightarrow 12, \text { as } h \rightarrow 0 .
\end{aligned}
$$

It is also easy to see that, for $j$ fixed,

$$
\lambda_{j}(h) \rightarrow\left(\frac{\pi j}{L}\right)^{2}, \text { as } h \rightarrow 0
$$

Indeed,

$$
\lim _{h \rightarrow 0} \lambda_{j}(h)=2 \lim _{h \rightarrow 0} \frac{1-\cos (j \pi h / L)}{h^{2}}=\frac{j \pi}{L} \lim _{h \rightarrow 0} \frac{\sin (j \pi h / L)}{h}=\left(\frac{j \pi}{L}\right)^{2} .
$$

Let us now analyze the gap between the roots of the eigenvalues. We have

$$
\begin{aligned}
\sqrt{\lambda_{j+1}(h)}-\sqrt{\lambda_{j}(h)} & =\frac{\sqrt{6}}{h}\left[\left(\frac{1-\cos ((j+1) \pi h / L)}{2+\cos ((j+1) \pi h / L)}\right)^{1 / 2}-\left(\frac{1-\cos (j \pi h / L)}{2+\cos (j \pi h / L)}\right)^{1 / 2}\right] \\
& =\frac{\sqrt{6} \pi}{L}\left[\frac{1}{2}\left(\frac{2+\cos (\xi)}{1-\cos (\xi)}\right)^{1 / 2}\left(\frac{3 \operatorname{sen} \xi}{(2+\cos \xi)^{2}}\right)\right]=3 \sqrt{\frac{3}{2}} \frac{\pi}{L} \frac{(1+\cos \xi)^{1 / 2}}{(2+\cos \xi)^{3 / 2}} \\
& \geq \sqrt{\frac{3}{2}} \frac{\pi}{L}\left(\frac{1+\cos \xi}{2+\cos \xi}\right)^{1 / 2}
\end{aligned}
$$

for some $\xi \in[j \pi h / L,(j+1) \pi h / L]$. 
Assume we consider eigenvalues corresponding to the indexes $(j+1) h \leq \varepsilon L$ with $0<\varepsilon<1$. Then $\xi \leq \varepsilon \pi$ and therefore $\cos \xi \geq \cos (\varepsilon \pi)$. Going back to (3.18) we deduce the following Lemma:

Lemma 3.2. For any $0<\varepsilon<1$ the gap between the roots of consecutive eigenvalues associated to indexes such that

$$
(j+1) h \leq \varepsilon L
$$

satisfies

$$
\sqrt{\lambda_{j+1}(h)}-\sqrt{\lambda_{j}(h)} \geq \frac{\pi}{L}\left(\frac{3(1+\cos (\varepsilon \pi))}{2(2+\cos (\varepsilon \pi))}\right)^{1 / 2}
$$

Observe that the lower bound on the gap vanishes as $\varepsilon \rightarrow 1$. However, it converges to $\pi / L$ when $\varepsilon \rightarrow 0$. In this sense the results are the same as those obtained in the previous section for the finite-difference semidiscretization.

We have an analogous result to Lemma 2.1:

Lemma 3.3. For any eigenvector $\varphi$ with eigenvalue $\lambda$ of (3.9) the following identity holds:

$$
\sum_{j=0}^{N}\left|\frac{\varphi_{j}-\varphi_{j+1}}{h}\right|^{2}=\lambda \sum_{j=1}^{N}\left(\frac{2}{3}\left|\varphi_{j}\right|^{2}+\frac{1}{3} \varphi_{j} \varphi_{j+1}\right)=\lambda \sum_{j=1}^{N}\left[\frac{1}{3}\left|\varphi_{j}\right|^{2}+\frac{1}{6}\left|\varphi_{j}+\varphi_{j+1}\right|^{2}\right]
$$

Proof. Identity (3.21) can be derived easily multiplying in (3.9) by $\varphi_{j}$ and adding for $j=1, \cdots, N$. (Note that (3.21) can be read as $(A \varphi, \varphi)=\lambda(M \varphi, \varphi))$.

Let us now prove identity (3.10) in Lemma 3.1. By virtue of (1.16) in Lemma 1.1 we have

$$
h \sum_{j=0}^{N}\left|\frac{\varphi_{j+1}-\varphi_{j}}{h}\right|^{2}=\frac{2 L}{4-\rho h^{2}}\left|\frac{\varphi_{N}}{h}\right|^{2}
$$

with $\rho$ eigenvalue of the matrix $A$ associated to $\varphi$. Then,

$$
\rho=\frac{\lambda}{1+\frac{h^{2}}{6} \lambda}
$$

and so,

$$
\frac{2 L}{4-\rho h^{2}}=\frac{2+\frac{2 h^{2}}{6} \lambda}{4+\frac{4 h^{2}}{6} \lambda-\lambda h^{2}} L=\frac{6+h^{2} \lambda}{12-\lambda h^{2}} L .
$$

As an immediate consequence of the identity (3.10) and (3.16) we deduce that Theorem 3.1 holds.

\subsection{Boundary observability of the semi-discrete wave equation}

The main goal of this section is to prove Theorem 3.2. To do that we first prove some basic identities.

Lemma 3.4. (Conservation of energy) For any $h>0$ and any solution of (3.1) it follows that

$$
E_{h}(t)=E_{h}(0), \quad \forall 0<t<T .
$$


Proof. Multiplying in (3.1) by $u_{j}^{\prime}$ we deduce that

$$
\sum_{j=1}^{N}\left(\frac{2}{3} u_{j}^{\prime \prime}+\frac{1}{6} u_{j+1}^{\prime \prime}+\frac{1}{6} u_{j-1}^{\prime \prime}\right) u_{j}^{\prime}=\sum_{j=1}^{N} \frac{\left(u_{j+1}+u_{j-1}-2 u_{j}\right)}{h^{2}} u_{j}^{\prime} .
$$

The right hand side term of (3.23) can be treated as in section 2. This yields

$$
\sum_{j=1}^{N}\left(\frac{u_{j+1}+u_{j-1}-2 u_{j}}{h^{2}}\right) u_{j}^{\prime}=\frac{1}{2} \frac{d}{d t} \sum_{j=0}^{N}\left|\frac{u_{j}-u_{j+1}}{h}\right|^{2} .
$$

The left hand side term can be handeled as follows:

$$
\begin{aligned}
\sum_{j=1}^{N}\left(\frac{2}{3} u_{j}^{\prime \prime}+\frac{1}{6} u_{j+1}^{\prime \prime}+\frac{1}{6} u_{j-1}^{\prime \prime}\right) u_{j}^{\prime} & =\frac{2}{3} \sum_{j=1}^{N} u_{j}^{\prime \prime} u_{j}^{\prime}+\frac{1}{6} \sum_{j=1}^{N}\left(u_{j+1}^{\prime \prime} u_{j}^{\prime}+u_{j-1}^{\prime \prime} u_{j}^{\prime}\right) \\
& =\frac{1}{3} \frac{d}{d t} \sum_{j=1}^{N}\left|u_{j}^{\prime}\right|^{2}+\frac{1}{6} \frac{d}{d t} \sum_{j=1}^{N} u_{j}^{\prime} u_{j+1}^{\prime} \\
& =\frac{1}{6} \frac{d}{d t} \sum_{j=1}^{N}\left|u_{j}^{\prime}\right|^{2}+\frac{1}{12} \frac{d}{d t} \sum_{j=1}^{N}\left|u_{j}^{\prime}+u_{j+1}^{\prime}\right|^{2}
\end{aligned}
$$

Combining (3.23-3.25) we deduce that $\frac{d E_{h}(t)}{d t}=0$ which is equivalent to (3.22).

Lemma 3.5. For any $h>0$ and any solution $u$ of (3.1) the following identity holds:

$$
T E_{h}(0)-\frac{h}{12} \sum_{j=0}^{N} \int_{0}^{T}\left|u_{j}^{\prime}-u_{j+1}^{\prime}\right|^{2} d t+\left.X_{h}(t)\right|_{0} ^{T}=\frac{L}{2} \int_{0}^{T}\left[\left|\frac{u_{N}(t)}{h}\right|^{2}+\frac{\left|u_{N}^{\prime}(t)\right|^{2}}{6}\right] d t
$$

with

$$
X_{h}(t)=h \sum_{j=1}^{N} j\left(u_{j+1}-u_{j-1}\right)\left(\frac{1}{3} u_{j}^{\prime}+\frac{1}{12} u_{j+1}^{\prime}+\frac{1}{12} u_{j-1}^{\prime}\right)
$$

Proof. We multiply in (3.1) by $j\left(u_{j+1}-u_{j-1}\right) / 2$. It follows that

$$
\begin{aligned}
& \sum_{j=1}^{N} \int_{0}^{T}\left[\frac{2}{3} u_{j}^{\prime \prime}+\frac{1}{6} u_{j+1}^{\prime \prime}+\frac{1}{6} u_{j-1}^{\prime \prime}\right] j\left(\frac{u_{j+1}-u_{j-1}}{2}\right) d t \\
& -\sum_{j=1}^{N} \int_{0}^{T}\left[\frac{u_{j+1}+u_{j-1}-2 u_{j}}{h^{2}}\right] j\left(\frac{u_{j+1}-u_{j-1}}{2}\right) d t=I_{1}-I_{2}=0 .
\end{aligned}
$$

Let us develop the two terms in (3.28). The second one $I_{2}$ can be treated as in the proof of Lemma 2.2. It follows that

$$
I_{2}=-\frac{1}{2} \sum_{j=0}^{N} \int_{0}^{T}\left|\frac{u_{j}-u_{j+1}}{h}\right|^{2} d t+\frac{(N+1)}{2} \int_{0}^{T}\left|\frac{u_{N}(t)}{h}\right|^{2} d t
$$


On the other hand, in view of (2.33),

$$
\begin{aligned}
I_{1}= & \sum_{j=1}^{N} \int_{0}^{T}\left[\frac{2}{3} u_{j}^{\prime \prime}+\frac{1}{6} u_{j+1}^{\prime \prime}+\frac{1}{6} u_{j-1}^{\prime \prime}\right] j \frac{\left(u_{j+1}-u_{j-1}\right)}{2} d t \\
= & \frac{1}{3} \sum_{j=1}^{N} \int_{0}^{T} u_{j}^{\prime} u_{j+1}^{\prime} d t+\left.\frac{2}{3} \sum_{j=1}^{N} u_{j}^{\prime} j \frac{\left(u_{j+1}-u_{j-1}\right)}{2}\right|_{0} ^{T} \\
& +\frac{1}{12} \sum_{j=1}^{N} \int_{0}^{T}\left(u_{j+1}^{\prime \prime}+u_{j-1}^{\prime \prime}\right) j\left(u_{j+1}-u_{j-1}\right) d t \\
= & \frac{1}{3} \sum_{j=1}^{N} \int_{0}^{T} u_{j}^{\prime} u_{j+1}^{\prime} d t+\left.\frac{1}{3} \sum_{j=1}^{N} u_{j}^{\prime} j\left(u_{j+1}-u_{j-1}\right)\right|_{0} ^{T} \\
& -\frac{1}{12} \sum_{j=1}^{N} \int_{0}^{T} j\left(\left|u_{j+1}^{\prime}\right|^{2}-\left|u_{j-1}^{\prime}\right|^{2}\right) d t+\left.\frac{1}{12} \sum_{j=1}^{N}\left(u_{j+1}^{\prime}+u_{j-1}^{\prime}\right) j\left(u_{j+1}-u_{j-1}\right)\right|_{0} ^{T} \\
& \frac{1}{3} \sum_{j=1}^{N} \int_{0}^{T} u_{j}^{\prime} u_{j+1}^{\prime} d t+\frac{1}{6} \sum_{j=1}^{N} \int_{0}^{T}\left|u_{j}^{\prime}\right|^{2} d t-\frac{N+1}{12} \int_{0}^{T}\left|u_{N}^{\prime}(t)\right|^{2} d t \\
& +\left.\left.\sum_{j=1}^{N}\right|_{j} ^{T}\left(u_{j+1}-u_{j-1}\right)\left[\frac{1}{3} u_{j}^{\prime}+\frac{1}{12} u_{j+1}^{\prime}+\frac{1}{12} u_{j-1}^{\prime}\right]\right|_{0} .
\end{aligned}
$$

Combining (3.28-3.30) we deduce that

$$
\begin{aligned}
\frac{L}{2} \int_{0}^{T}\left[\left|\frac{u_{N}(t)}{h}\right|^{2}+\frac{\left|u_{N}^{\prime}(t)\right|^{2}}{6}\right] d t= & \frac{h}{2} \sum_{j=0}^{N} \int_{0}^{T}\left|\frac{u_{j}-u_{j+1}}{h}\right|^{2} d t+\frac{h}{3} \sum_{j=1}^{N} \int_{0}^{T} u_{j}^{\prime} u_{j+1}^{\prime} d t \\
& +\frac{h}{6} \sum_{j=1}^{N} \int_{0}^{T}\left|u_{j}^{\prime}\right|^{2} d t+\left.X_{n}(t)\right|_{0} ^{T} \\
= & T E_{h}(0)+\frac{h}{3} \sum_{j=0}^{N} \int_{0}^{T}\left[u_{j}^{\prime} u_{j+1}^{\prime}-\frac{\left|u_{j}^{\prime}+u_{j+1}^{\prime}\right|^{2}}{4}\right] d t+\left.X_{h}(t)\right|_{0} ^{T}
\end{aligned}
$$

Finally we observe that

$$
\sum_{j=0}^{N} \int_{0}^{T}\left[u_{j}^{\prime} u_{j+1}^{\prime}-\frac{\left|u_{j}^{\prime}+u_{j+1}^{\prime}\right|^{2}}{4}\right] d t=-\sum_{j=0}^{N} \int_{0}^{T} \frac{\left|u_{j}^{\prime}-u_{j+1}^{\prime}\right|^{2}}{4} d t
$$

Combining (3.31) and (3.32) we obtain (3.26). 
Lemma 3.6. (Equipartition of energy) For any $h>0$ and any solution $u$ of (3.1) the following identity holds:

$$
h \int_{0}^{T} \sum_{j=0}^{N}\left|\frac{u_{j+1}-u_{j}}{h}\right|^{2} d t=h \int_{0}^{T} \sum_{j=0}^{N}\left[\frac{1}{3}\left|u_{j}^{\prime}\right|^{2}+\frac{1}{6}\left|u_{j}^{\prime}+u_{j+1}^{\prime}\right|^{2}\right] d t-\left.Y_{h}(t)\right|_{0} ^{T}
$$

with

$$
Y_{h}(t)=h \sum_{j=1}^{N}\left(\frac{2}{3} u_{j}^{\prime}+\frac{1}{6} u_{j+1}^{\prime}+\frac{1}{6} u_{j-1}^{\prime}\right) u_{j}
$$

Proof. We multiply in (3.1) by $u_{j}$. It follows that

$$
h \sum_{j=1}^{N} \int_{0}^{T}\left(\frac{2}{3} u_{j}^{\prime \prime}+\frac{1}{6} u_{j+1}^{\prime \prime}+\frac{1}{6} u_{j-1}^{\prime \prime}\right) u_{j} d t=h \sum_{j=1}^{N} \int_{0}^{T}\left[\frac{u_{j+1}+u_{j-1}-2 u_{j}}{h^{2}}\right] u_{j} d t .
$$

We have

$$
\begin{aligned}
h \sum_{j=1}^{N} \int_{0}^{T}\left(\frac{2}{3} u_{j}^{\prime \prime}+\frac{1}{6} u_{j+1}^{\prime \prime}+\frac{1}{6} u_{j-1}^{\prime \prime}\right) u_{j} d t= & \left.h \sum_{j=1}^{N}\left(\frac{2}{3} u_{j}^{\prime}+\frac{1}{6} u_{j+1}^{\prime}+\frac{1}{6} u_{j-1}^{\prime}\right) u_{j}\right|_{0} ^{T} \\
& -h \sum_{j=1}^{N} \int_{0}^{T}\left(\frac{2}{3} u_{j}^{\prime}+\frac{1}{6} u_{j+1}^{\prime}+\frac{1}{6} u_{j-1}^{\prime}\right) u_{j}^{\prime} d t \\
= & \left.Y_{h}(t)\right|_{0} ^{T}-h \sum_{j=1}^{N} \int_{0}^{T}\left(\frac{2}{3}\left|u_{j}^{\prime}\right|^{2}+\frac{1}{3} u_{j}^{\prime} u_{j+1}^{\prime}\right) d t \\
= & \left.Y_{h}(t)\right|_{0} ^{T}-h \sum_{j=1}^{N} \int_{0}^{T}\left(\frac{1}{3}\left|u_{j}^{\prime}\right|^{2}+\frac{1}{6}\left|u_{j}^{\prime}+u_{j+1}^{\prime}\right|^{2}\right) d t
\end{aligned}
$$

On the other hand,

$$
h \sum_{j=1}^{N} \int_{0}^{T}\left(\frac{u_{j+1}-u_{j-1}-2 u_{j}}{h^{2}}\right) u_{j} d t=-h \sum_{j=0}^{N} \int_{0}^{T}\left|\frac{u_{j}-u_{j+1}}{h}\right|^{2} d t .
$$

Combining (3.35-3.37) we deduce (3.33).

Let us now estimate the term $\sum_{j=0}^{N} \int_{0}^{T}\left|u_{j}^{\prime}-u_{j+1}^{\prime}\right|^{2} d t$ in (3.26). Let $\Lambda$ be the largest eigenvalue entering in the Fourier development of the solution $u$ of $(3.1)$, i.e.,

$$
u=\sum_{\left|\mu_{k}\right| \leq \sqrt{\Lambda}} a_{k} e^{i \mu_{k} t} \varphi^{k}
$$

with $\mu_{k}=\sqrt{\lambda_{k}}$ for $k>0$ and $\mu_{k}=-\mu_{-k}$ when $k<0$. Therefore

$$
u^{\prime}=i \sum_{\left|\mu_{k}\right| \leq \sqrt{\Lambda}} a_{k} \mu_{k} e^{i \mu_{k} t} \varphi^{k} .
$$


Thus, using Lemmas 2.1 and 3.3 we have:

$$
\begin{aligned}
\sum_{j=0}^{N}\left|u_{j}^{\prime}-u_{j+1}^{\prime}\right|^{2}= & \sum_{j=0}^{N}\left|\sum_{\left|\mu_{k}\right| \leq \sqrt{\Lambda}} a_{k} \mu_{k} e^{i \mu_{k} t}\left(\varphi_{k, j}-\varphi_{k, j+1}\right)\right|^{2} \\
= & \sum_{j=0}^{N} \sum_{\left|\mu_{k}\right| \leq \sqrt{\Lambda}}\left|a_{k}\right|^{2} \mu_{k}^{2}\left|\varphi_{k, j}-\varphi_{k, j+1}\right|^{2} \\
& +\sum_{j=0}^{N} \sum_{\substack{\left|\mu_{k}\right| \leq \sqrt{\Lambda} \\
\left|\mu_{\ell}\right| \leq \sqrt{\Lambda} \\
\mu_{k} \neq \mu_{\ell}}} \mu_{k} \mu_{\ell} a_{k} \bar{a}_{\ell} e^{i\left(\mu_{k}-\mu_{\ell}\right) t}\left(\varphi_{k, j}-\varphi_{k, j+1}\right)\left(\varphi_{\ell, j}-\varphi_{\ell, j+1}\right) \\
= & \left.\sum_{j=0}^{N} \sum_{\substack{\left|\mu_{k}\right| \leq \sqrt{\Lambda} \\
=}} \lambda_{k}\left|a_{k} \sum_{j=1}^{N} \sum_{\left|\mu_{k}\right| \leq \sqrt{\Lambda}} \lambda_{k}^{2}\right| a_{k}\right|^{2}\left(\frac{1}{3}\left|\varphi_{k, j}\right|^{2}+\frac{1}{6}\left(\varphi_{k, j}+\varphi_{k, j+1}\right)^{2}\right) \\
\leq & h^{2} \Lambda \sum_{j=1}^{N} \sum_{\left|\mu_{k}\right| \leq \sqrt{\Lambda}} \lambda_{k}\left|a_{k}\right|^{2}\left(\frac{1}{3}\left|\varphi_{k, j}\right|^{2}+\frac{1}{6}\left(\varphi_{k, j}+\varphi_{k, j+1}\right)^{2}\right) \\
= & h^{2} \Lambda \sum_{j=0}^{N}\left[\frac{1}{3}\left|u_{j}^{\prime}\right|^{2}+\frac{1}{6}\left|u_{j}^{\prime}+u_{j+1}^{\prime}\right|^{2}\right] .
\end{aligned}
$$

In the last step we have used the fact that

$$
\sum_{j=0}^{N}\left[\frac{1}{3} \varphi_{k, j} \varphi_{\ell, j}+\frac{1}{6}\left(\varphi_{k, j}+\varphi_{k, j+1}\right)\left(\varphi_{\ell, j}+\varphi_{\ell, j+1}\right)\right]=0
$$

if $\varphi_{k}$ and $\varphi_{\ell}$ are eigenvectors associated to eigenvalues $\lambda_{k} \neq \lambda_{\ell}$, which is a consequence of (2.6).

On the other hand, combining the conservation of energy identity (3.22) and the equipartition of energy (3.33) we deduce that

$$
h \int_{0}^{T} \sum_{j=0}^{N}\left(\frac{1}{3}\left|u_{j}^{\prime}\right|^{2}+\frac{1}{6}\left|u_{j}^{\prime}+u_{j+1}^{\prime}\right|^{2}\right) d t=T E(0)+\left.\frac{Y_{h}(t)}{2}\right|_{0} ^{T} .
$$

Combining (3.31-3.32) with (3.39-3.40) we deduce that

$$
T\left(1-\frac{\Lambda h^{2}}{12}\right) E(0)+\left.Z_{h}(t)\right|_{0} ^{T} \leq \frac{L}{2} \int_{0}^{T}\left[\left|\frac{u_{N}(t)}{h}\right|^{2}+\frac{\left|u_{N}^{\prime}(t)\right|^{2}}{6}\right] d t
$$

with

$$
Z_{h}(t)=X_{h}(t)-\frac{\Lambda h^{2}}{24} Y_{h}(t)
$$


The following holds:

Lemma 3.7. For any $h>0$ and any solution $u$ of (3.1) it follows that

$$
\left|Z_{h}(t)\right| \leq \sqrt{L^{2}-\frac{\Lambda h^{4}}{48}+\frac{3 \Lambda h^{2}}{16 \lambda_{1}}}, \quad \forall 0<t<T .
$$

Proof. To simplify the notation we do not make explicit the dependence on $t$. We have

$$
\begin{aligned}
Z_{h}(t) & =X_{h}(t)-\frac{\Lambda h^{2}}{24} Y_{h}(t) \\
& =h \sum_{j=1}^{N}\left(j \frac{\left(u_{j+1}-u_{j}\right)}{2}-\frac{\Lambda h^{2}}{24} u_{j}\right)\left(\frac{2}{3} u_{j}^{\prime}+\frac{1}{6} u_{j+1}^{\prime}+\frac{1}{6} u_{j-1}^{\prime}\right) \\
& =h \sum_{i, j=1}^{N} m_{i j} a_{i} b_{j}
\end{aligned}
$$

with

and $m_{i j}$ the entries of matrix $M$.

$$
a_{i}=i \frac{\left(u_{i+1}-u_{i}\right)}{2}-\frac{\Lambda h^{2}}{24} u_{i} ; b_{i}=u_{i}^{\prime}
$$

Therefore

$$
\left|Z_{h}\right| \leq\left(h \sum_{i, j=1}^{N} m_{i j} a_{i} a_{j}\right)^{1 / 2}\left(h \sum_{i, j=1}^{N} m_{i j} b_{i} b_{j}\right)^{1 / 2}
$$

On the other hand,

$$
h \sum_{i, j=1}^{N} m_{i j} b_{i} b_{j}=h \sum_{j=1}^{N}\left[\frac{2}{3}\left|u_{j}^{\prime}\right|^{2}+\frac{1}{6}\left(u_{j+1}^{\prime}+u_{j-1}^{\prime}\right) u_{j}^{\prime}\right]=h\left[\sum_{j=0}^{N} \frac{1}{3}\left|u_{j}^{\prime}\right|^{2}+\frac{1}{6}\left|u_{j}^{\prime}+u_{j+1}^{\prime}\right|^{2}\right]
$$

and

$$
\begin{aligned}
h \sum_{i, j=1}^{N} m_{i j} a_{i} a_{j} & \leq h \sum_{j=1}^{N}\left|a_{i}\right|^{2}=\frac{h}{4} \sum_{j=1}^{N}\left[j\left(u_{j+1}-u_{j-1}\right)-\frac{\Lambda h^{2}}{12} u_{j}\right]^{2} \\
& \leq \frac{h}{4} \sum_{j=1}^{N}\left[2 j^{2}\left|u_{j+1}-u_{j}\right|^{2}+2 j^{2}\left|u_{j}-u_{j-1}\right|^{2}+\frac{\Lambda^{2} h^{4}}{144} u_{j}^{2}-\frac{\Lambda h^{2}}{6} j u_{j}\left(u_{j+1}-u_{j-1}\right)\right] \\
& \leq L^{2} h \sum_{j=0}^{N}\left|\frac{u_{j+1}-u_{j}}{h}\right|^{2}+\frac{\Lambda^{2} h^{5}}{576} \sum_{j=1}^{N}\left|u_{j}\right|^{2}+\frac{\Lambda h^{3}}{24} \sum_{j=1}^{N} u_{j} u_{j+1} \\
& \leq L^{2} h \sum_{j=0}^{N}\left|\frac{u_{j+1}-u_{j}}{h}\right|^{2}-\frac{\eta h^{2}}{2} h \sum_{j=0}^{N}\left|\frac{u_{j+1}-u_{j}}{h}\right|^{2}+\left(\eta^{2}+\eta\right) h \sum_{j=1}^{N}\left|u_{j}\right|^{2} \\
& \leq\left(L^{2}-\frac{\eta h^{2}}{2}+\frac{3\left(\eta^{2}+\eta\right)}{\lambda_{1}}\right) h \sum_{j=0}^{N}\left|\frac{u_{j+1}-u_{j}}{h}\right|^{2} \leq\left(L^{2}-\frac{\eta h^{2}}{2}+\frac{9 \eta}{2 \lambda_{1}}\right) h \sum_{j=0}^{N}\left|\frac{u_{j+1}-u_{j}}{h}\right|^{2}
\end{aligned}
$$


We have used the notation $\eta=\Lambda h^{2} / 24$, the fact that $\eta \leq 1 / 2$ and also that

$$
\frac{1}{3} h \sum_{j=1}^{N}\left|u_{j}\right|^{2} \leq \frac{1}{\lambda_{1}} h \sum_{j=0}^{N}\left|\frac{u_{j+1}-u_{j}}{h}\right|^{2}
$$

which is a consequence of (3.21).

Combining (3.44-3.46) we deduce that

$$
\begin{aligned}
\left|Z_{h}\right| & \leq\left[h \sum_{j=1}^{N}\left(\frac{1}{3}\left|u_{j}^{\prime}\right|^{2}+\frac{1}{6}\left|u_{j}^{\prime}+u_{j+1}^{\prime}\right|^{2}\right)\right]^{1 / 2}\left[\left(L^{2}-\frac{\eta h^{2}}{2}+\frac{9 \eta}{2 \lambda_{1}}\right) h \sum_{j=0}^{N}\left|\frac{u_{j+1}-u_{j}}{h}\right|^{2}\right]^{1 / 2} \\
& \leq \sqrt{L^{2}-\frac{\eta h^{2}}{2}+\frac{9 \eta}{2 \lambda_{1}}} E_{h}(0)
\end{aligned}
$$

which is equivalent to (3.43).

Let us now set $\Lambda=\gamma / h^{2}$. Combining (3.41) and (3.43) we obtain

$$
\left[T(1-\gamma / 12)-2 \sqrt{L^{2}-\frac{\gamma h^{2}}{48}+\frac{3 \gamma}{16 \lambda_{1}}}\right] E_{h}(0) \leq \frac{L}{2} \int_{0}^{T}\left[\left|\frac{u_{N}(t)}{h}\right|^{2}+\frac{\left|u_{N}^{\prime}(t)\right|^{2}}{6}\right] d t .
$$

Therefore, the statement of Theorem 3.2 holds with

$$
T(\gamma)=\frac{2 \sqrt{L^{2}+\frac{\gamma}{16}\left(\frac{3}{\lambda_{1}}-\frac{h^{2}}{3}\right)}}{1-\gamma / 12}
$$

and

$$
C(T, \gamma)=\frac{L}{2\left(T(1-\gamma / 12)-2 \sqrt{L^{2}+\frac{\gamma}{16}\left(\frac{3}{\lambda_{1}}-\frac{h^{2}}{3}\right)}\right)} .
$$

\subsection{Boundary observability of the semi-discrete wave equation: improved estimates}

The goal of this section is to get rid of the term $\int_{0}^{T}\left|u_{N}^{\prime}(t)\right|^{2} d t$ on the right hand side of (3.14).

Note that the solution $u$ of (3.1) can be written as

$$
u=\sum a_{k} e^{i \mu_{k} t} \varphi^{k}
$$

and therefore

$$
u_{N}^{\prime}(t)=i \sum a_{k} \mu_{k} e^{i \mu_{k} t} \varphi_{k, N} .
$$

Thus

$$
\int_{0}^{T}\left|u_{N}^{\prime}(t)\right|^{2} d t=\int_{0}^{T}\left|\sum a_{k} \mu_{k} e^{i \mu_{k} t} \varphi_{k, N}\right|^{2} d t .
$$

We now restrict our analysis to the solutions in the class $\mathcal{C}_{h}(\gamma)$ that are generated by the eigenvectors associated to eigenvalues

$$
\lambda \leq \gamma / h^{2}
$$


with $0<\gamma<12$. Restriction (3.49) is equivalent to

$$
(j+1) h \leq \varepsilon(\gamma) L
$$

for a suitable $0<\varepsilon(\gamma)<1$. We shall return later to the explicit computation of $\varepsilon(\gamma)$.

Then, according (3.20) the gap between the roots of consecutive eigenvalues in the range (3.49) is given by

$$
\frac{\pi}{L}\left(\frac{3(1+\cos (\varepsilon \pi))}{2(2+\cos (\varepsilon \pi))}\right)^{1 / 2} .
$$

Then, by means of Ingham's inequality [7] we deduce that, provided,

$$
T>2 L\left(\frac{2(2+\cos (\varepsilon \pi))}{3(1+\cos (\varepsilon \pi))}\right)^{1 / 2}
$$

it follows that

$$
\begin{aligned}
\int_{0}^{T}\left|\sum_{\left|\mu_{k}\right| \leq \sqrt{\gamma} / h} a_{k} \mu_{k} e^{i \mu_{k} t} \varphi_{k, N}\right|^{2} & \leq C_{2}(T, \varepsilon(\gamma)) \sum_{\left|\mu_{k}\right| \leq \sqrt{\gamma} / h}\left|a_{k}\right|^{2} \lambda_{k}\left|\varphi_{k, N}\right|^{2} \\
& \leq C_{2}(T, \varepsilon(\gamma)) \gamma \sum_{\left|\mu_{k}\right| \leq \sqrt{\gamma} / h}\left|a_{k}\right|^{2}\left|\frac{\varphi_{k, N}}{h}\right|^{2} \\
& \leq \frac{C_{2}(T, \varepsilon(\gamma)) \gamma}{C_{1}(T, \varepsilon(\gamma))} \int_{0}^{T}\left|\sum_{\left|\mu_{k}\right| \leq \sqrt{\gamma} / h} a_{k} e^{i \mu_{k} t} \frac{\varphi_{k, N}}{h}\right|^{2} d t \\
& =\frac{\gamma C_{2}(T, \varepsilon(\gamma))}{C_{1}(T, \varepsilon(\gamma))} \int_{0}^{T}\left|\frac{u_{N}(t)}{h}\right|^{2} d t .
\end{aligned}
$$

Combining Theorem 3.2 and (3.53) it follows that:

Theorem 3.3. For any $0<\gamma<12$ and

$$
T>\max \left(T(\gamma), 2 L\left(\frac{2(2+\cos (\varepsilon \pi))}{3(1+\cos (\varepsilon \pi))}\right)^{1 / 2}\right)
$$

it follows that

$$
E_{h}(0) \leq\left[C(T, \gamma)+\frac{\gamma C_{2}(T, \varepsilon(\gamma))}{6 C_{1}(T, \varepsilon(\gamma))}\right] \int_{0}^{T}\left|\frac{u_{N}(t)}{h}\right|^{2} d t
$$

for any solution $u$ of $(3.1)$ in the class $\mathcal{C}_{h}(\gamma)$.

Remark. The time of observability in Theorem 3.3 is the maximum between the time $T(\gamma)$ in Theorem 3.2 and the time (3.52) needed to apply Ingham's inequality to the eigenvalues corresponding to the class of solutions $\mathcal{C}_{h}(\gamma)$. The observability constant in (3.55) is the addition of the constant $C(T, \gamma)$ of Theorem 3.2 and the constant needed to absorve the term $\int_{0}^{T}\left|u_{N}^{\prime}(t)\right|^{2} d t$ by Ingham's inequality as in (3.53).

Note that $\varepsilon(\gamma) \rightarrow 0$ as $\gamma \rightarrow 0$ while the constants $C_{1}(T, \varepsilon)$ and $C_{2}(T, \varepsilon)$ in Ingham's inequality converge to 1 . Thus, according to Theorem 3.2, the observability constant in (3.55) converges to $L /[2(T-2 L)]$ as $\gamma \rightarrow 0$. 
Observe that an inequality of the form (3.55) can also be obtained directly by Ingham's inequality. However we have preferred to obtain the weaker form of observability inequality in Theorem 3.2 by multipliers since this method can be more easily adapted to other situations.

Let us now compute $\varepsilon(\gamma)$. According to the explicit value of the eigenvalues obtained in (3.15) we have to estimate $j$ so that

$$
6(1-\cos ((j+1) \pi h / L)) \leq \gamma(2+\cos ((j+1) \pi h / L)),
$$

or, taking into account that $(j+1) h \leq \varepsilon L$, we have to estimate $\varepsilon>0$ such that

$$
6(1-\cos (\varepsilon \pi)) \leq \gamma(2+\cos (\varepsilon \pi))
$$

or, equivalently,

$$
\begin{gathered}
6-2 \gamma \leq(\gamma+6) \cos (\varepsilon \pi), \\
\varepsilon(\gamma)=\frac{1}{\pi} \arccos \left(\frac{6-2 \gamma}{\gamma+6}\right) .
\end{gathered}
$$$$
\text { i.e., }
$$

Obviously $\varepsilon(\gamma) \rightarrow 0$ as $\gamma \rightarrow 0$ and $\varepsilon(\gamma) \rightarrow 1$ as $\gamma \rightarrow 12$.

According to (3.52) and (3.56) the time needed to apply Ingham's inequality is:

$$
\frac{2 L}{((1+\cos (\varepsilon(\gamma) \pi)) / 2)^{1 / 2}}=2 L\left(\frac{12+2 \gamma}{12-\gamma}\right)^{1 / 2} .
$$

\section{Comparison}

In this section we briefly compare the observability results we have obtained for the finite-difference and the finite-element semi-discretizations.

We focus on inequalities of the form

$$
E_{h}(0) \leq C \int_{0}^{T}\left|\frac{u_{N}(t)}{h}\right|^{2} d t
$$

both, for solutions of system (1.6) and (3.1).

We consider only the estimates of the form (4.1) that may be obtained directly by means of Ingham's inequalities. For, we consider solutions of (1.6) and (3.1) generated by the eigenvectors with indexes

$$
1 \leq j,(j+1) h \leq \varepsilon L
$$

with $0<\varepsilon<1$, for which the observability inequalities are known to be uniform as $h \rightarrow 0$. Thus, we are considering subspaces of solutions of dimension

$$
d=\left[\frac{\varepsilon L}{h}\right]-1
$$

Let us first compare the observability time that is required in both cases. We recall that the minimal observability time depends on the gap between the roots of consecutive eigenvalues. In the case of finite-differences, as shown in Lemma 2.6, the gap is of the order of

$$
\pi \cos (\pi \varepsilon / 2) / L
$$


This leads to an observability time

$$
T_{1}=2 L / \cos (\pi \varepsilon / 2)
$$

In the case of the finite-element approximation the gap is (see (3.18))

$$
\frac{\pi}{L}\left(\frac{3(1+\cos (\varepsilon \pi))}{2(2+\cos (\varepsilon \pi))}\right)^{1 / 2}
$$

and the observability time

$$
T_{2}=2 L\left(\frac{2(2+\cos (\varepsilon \pi))}{3(1+\cos (\varepsilon \pi))}\right)^{1 / 2}
$$

Let us see that $T_{1}>T_{2}$. Indeed,

$$
T_{1}>T_{2} \Leftrightarrow \cos ^{2}(\pi \varepsilon / 2)<\frac{3(1+\cos (\varepsilon \pi))}{2(2+\cos (\varepsilon \pi))}
$$

or in other words, if and only if,

which is obviously true.

$$
(2+\cos (\varepsilon \pi))<3
$$

This indicates that the observability time for the finite-difference semi-discretization is larger than for the finite-element one.

This is in agreement with the analysis of the phase velocity of the semi-discretizations of the wave equation in [12], Section 2.10.

The authors acknowledge C. Fabre for fruitful comments on the first version of this paper.

\section{REFERENCES}

[1] M. Avellaneda, C. Bardos and J. Rauch, Contrôlabilité exacte, homogéneisation et localisation d'ondes dans un milieu nonhomogène. Asymptotic Analysis 5 (1992) 481-494.

[2] C.Castro and E. Zuazua, Contrôle de l'équation des ondes à densité rapidement oscillante à une dimension d'espace. $C$. $R$. Acad. Sci. Paris 324 (1997) 1237-1242.

[3] R. Glowinski, Ensuring Well-Posedness by Analogy; Stokes Problem and Boundary Control for the Wave Equation. J. Comput. Phys. 103 (1992) 189-221.

[4] R. Glowinski, C.H. Li and J.L. Lions, A numerical approach to the exact boundary controllability of the wave equation. (I). Dirichlet Controls: Description of the numerical methods. Jap. J. Appl. Math. 7 (1990) 1-76.

[5] R. Glowinski and J.L. Lions, Exact and approximate controllability for distributed parameter systems. Acta Numerica (1996) $159-333$.

[6] J.A. Infante and E. Zuazua, Boundary observability for the space-discretizations of the $1-d$ wave equation. C. R. Acad. Sci. Paris 326 (1998) 713-718.

[7] A.E. Ingham, Some trigonometrical inequalities with applications to the theory of series. Mathematische Zeitschrift 41 (1936) $367-379$.

[8] E. Isaacson and H.B. Keller, Analysis of Numerical Methods. John Wiley \& Sons (1966).

[9] V. Komornik, Exact controllability and stabilization. The multiplier method. John Wiley \& Sons, Masson (1994).

[10] E.B. Lee and L. Markus, Foundations of Optimal Control Theory, The SIAM Series in Applied Mathematics. John Wiley \& Sons (1967).

[11] J.L. Lions, Contrôlabilité exacte, stabilisation et perturbations de systèmes distribués. Tome 1. Contrôlabilité exacte. Masson, RMA8 (1988).

[12] R. Vichnevetsky and J.B. Bowles, Fourier analysis of numerical approximations of hyperbolic equations. SIAM, Philadelphia (1982). 